TITLE:

\title{
Spectral function of a fermion coupled with a massive vector boson at finite temperature in a gauge invariant formalism
}

\section{AUTHOR(S):}

Satow, Daisuke; Hidaka, Yoshimasa; Kunihiro, Teiji

\section{CITATION:}

Satow, Daisuke ...[et al]. Spectral function of a fermion coupled with a massive vector boson at finite temperature in a gauge invariant formalism. Physical Review D 2011, 83(4): 045017.

\section{ISSUE DATE:}

2011-02

URL:

http://hdl.handle.net/2433/139532

RIGHT:

C) 2011 American Physical Society 
PHYSICAL REVIEW D 83, 045017 (2011)

\title{
Spectral function of a fermion coupled with a massive vector boson at finite temperature in a gauge invariant formalism
}

\author{
Daisuke Satow, ${ }^{*}$ Yoshimasa Hidaka, ${ }^{\dagger}$ and Teiji Kunihiro ${ }^{\ddagger}$ \\ Department of Physics, Faculty of Science, Kyoto University, Kitashirakawa Oiwakecho, Sakyo-ku, Kyoto 606-8502, Japan \\ (Received 7 December 2010; published 18 February 2011)
}

\begin{abstract}
We investigate spectral properties of a fermion coupled with a massive gauge boson with a mass $m$ at finite temperature $(T)$ in the perturbation theory. The massive gauge boson is introduced as a $U(1)$ gauge boson in the Stueckelberg formalism with a gauge parameter $\alpha$. We find that the fermion spectral function has a three-peak structure for $T \sim m$ irrespective of the choice of the gauge parameter, while it tends to have one faint peak at the origin and two peaks corresponding to the normal fermion and antiplasmino excitations familiar in QED in the hard thermal loop approximation for $T \gg m$. We show that our formalism successfully describe the fermion spectral function in the whole $T$ region with the correct high- $T$ limit except for the faint peak at the origin, although some care is needed for choice of the gauge parameter for $T \gg m$. We clarify that for $T \sim m$, the fermion pole is almost independent of the gauge parameter in the one-loop order, while for $T \gg m$, the one-loop analysis is valid only for $\alpha \ll 1 / g$ where $g$ is the fermion-boson coupling constant, implying that the one-loop analysis cannot be valid for large gauge parameters as in the unitary gauge.
\end{abstract}

DOI: 10.1103/PhysRevD.83.045017

PACS numbers: 11.10.Wx, 12.38.Mh

\section{INTRODUCTION}

It is well known that for extremely high temperature ( $T$ ) where the hard thermal loop (HTL) approximation in QED and QCD [1-4] is valid, a fermion (quark) coupled with thermally excited gauge fields (gluons) make collective excitations, i.e., the normal fermion (particle) and the antiplasmino excitation with distinct peaks in the fermion spectral function [4]; this feature obtained in the HTL approximation is also known to be gauge invariant in the sense that the fermion self-energy at one-loop order does not depend on gauge fixing [2]. As for the lower $T$ region, a possible change in the spectral properties of the quark in association with the chiral transition in QCD was investigated [5], using the Nambu-Jona-Lasinio model [6], and it is shown that the coupling with the chiral soft modes [7] makes the quark spectral function have distinct three peaks near but above the critical temperature of chiral transition. The appearance of such a novel spectral function at $T \sim m$ was later confirmed [8] for a massless fermion coupled with an elementary massive boson with a mass $m$, irrespective of the type of the massive boson. The mechanism for realizing the three-peak structure in the spectral function was also elucidated [8] in terms of the Landau damping owing to the collisions of the fermion with thermally excited bosons. ${ }^{1}$

Then one may naturally ask a question if the fermion spectral function at $T \sim m$ would smoothly connect with that at extremely high $T$, i.e., the HTL result in QED/QCD:

\footnotetext{
*d-sato@ruby.scphys.kyoto-u.ac.jp

†hidaka@ruby.scphys.kyoto-u.ac.jp

kunihiro@ruby.scphys.kyoto-u.ac.jp

${ }^{1}$ This feature where the three-peak structure arises at $T \sim m$ is not altered even for a massive fermion with a mass $m_{f}$ as long as $m_{f}$ is not too large compared with $m$ [9].
}

If this is not the case, it means that we do not have a unified understanding of the fermion spectral properties in the whole $T$ region. Partly to answer this question, we investigate the spectral properties of a fermion coupled with a massive vector boson introduced as a $U(1)$ gauge boson in the (generalized) Stueckelberg formalism with a gauge parameter $\alpha[10,11]$, and carefully examine their possible gauge dependence at $T \neq 0$, at the one-loop order as in [8]. Here the spectral properties include the number of the fermion poles, the pole position in the complex energy plane, and the spectral function in the momentum-energy plane. We are also interested in how the quasiparticle nature of the fermion is realized or destroyed by the coupling with a massive boson at finite $T$.

We find that the present formalism gives a valid description of the fermion coupled with a massive vector boson for the whole temperature $(T)$ region at one-loop order in a unified way; thereby we reveal the characteristics of the fermion spectral properties depending on the distinct $T$ regions, i.e., (I) $T \ll m$, (II) $T \sim m$, and (III) $T \gg m$. In particular, we shall show that the fermion spectral function certainly tends to have a three-peak structure for $T \sim m$ in the small momentum region with supports in the positive, zero, and negative energy regions.

The investigation of the possible gauge dependence turns out to be involved due to the appearance of a novel mass scale $\sqrt{\alpha} m$, inherent in the present formalism, as well as the boson mass $m$ and temperature $T$. One should remark here that the Proca formalism adopted in [8] is not adequate for this purpose, because this formalism corresponds to a specific gauge-fixing with $\alpha \rightarrow \infty$ (unitary gauge), and does not lead to the proper high- $T$ limit, or $m / T \rightarrow 0$, which should be the HTL approximation in QED at the one-loop level [4]. This is the reason why we 
have adopted the Stueckelberg formalism to describe the massive vector boson. We remark that although the pole position is gauge independent in the exact calculation [12], a gauge dependence of the fermion pole may appear in the perturbation theory at finite $T$ in general. Since the Proca formalism corresponding to the limit $\alpha \rightarrow \infty$ leads to a wrong high- $T$ limit, an adequate gauge-parameter region should exist in which the results in the perturbation theory hardly show gauge dependence: Indeed, we show that this is the case in the present work.

There are some physical situations to which the present analysis can have relevance since massive vector bosons exist or appear at finite $T$ in various physical systems. In $\mathrm{QCD}$, vector bosons or vector-bosonic modes, for example, may become a soft mode associated to the restoration of chiral symmetry at finite $T$ [13]. Moreover vector-bosonic modes might exist even in the deconfined and chiral symmetric phase in the vicinity of the critical temperature $T_{c}$. In the electroweak theory, neutrinos may modify their dispersion relations in a drastic way at high $T$ through scatterings with the weak bosons whose masses would change with $T$ [14]. We shall present the physical implication of the present results to various physical systems, including the above examples in the concluding remarks.

This paper is organized as follows. In Sec. II, we formulate the $U(1)$ gauge theory in which the gauge boson acquires finite mass. We perform a calculation of a fermion self-energy at finite temperature. In Sec. III, the numerical results of the fermion spectral properties are shown. In Sec. IV, we discuss the gauge dependence of the fermion pole appearing when $T \gg m$ in an analytic way. Section $\mathrm{V}$ is devoted to a summary and concluding remarks. In Appendix A, we briefly describe how the Abelian-Higgs model is reduced to the massive gauge theory in the Stueckelberg formalism. In Appendix B, we present detailed calculational procedures for the fermion self-energy in our model. Appendix $\mathrm{C}$ is devoted to making an order estimate of some terms appearing in the text.

\section{II. $U(1)$ GAUGE THEORY WITH MASSIVE GAUGE BOSON}

In this section, we formulate the $U(1)$ gauge theory with a massive gauge boson, and introduce a propagator and a spectral function at finite temperature in the imaginary time formalism $[15,16]$. We perform a calculation of the self-energy of a fermion coupled with a massive vector boson at one-loop order.

\section{A. General formalism}

First, we introduce a $U(1)$ gauge theory with a massive gauge boson. The gauge boson acquires a mass by the Higgs mechanism, keeping the gauge symmetry. The gauge theory is one way to construct a renormalizable quantum field theory with a massive vector boson. We employ the Stueckelberg formalism [10,11] proposed long ago, which is equivalent to the Abelian-Higgs model with a constant absolute value of the Higgs field [11,17]. This correspondence is reviewed in Appendix A. Then our Lagrangian reads

$$
\begin{aligned}
\mathcal{L}= & -\frac{1}{4} F_{\mu \nu} F^{\mu \nu}+\frac{1}{2} m^{2}\left(A_{\mu}-\frac{\partial_{\mu} B}{m}\right)\left(A^{\mu}-\frac{\partial^{\mu} B}{m}\right) \\
& +\bar{\psi}\left(i\left(\partial_{\mu}-i g A_{\mu}\right) \gamma^{\mu}\right) \psi+\mathcal{L}_{\mathrm{GF}},
\end{aligned}
$$

where $A_{\mu}, B$, and $\psi$ are a massive vector, a scalar, and a fermion field, respectively. The scalar field $B$ is called the Stueckelberg field, which corresponds to the phase of the Higgs field in the Abelian-Higgs model. $F_{\mu \nu}=\partial_{\mu} A_{\nu}-$ $\partial_{\nu} A_{\mu}$ is a field strength, $g$ the coupling constant, $m$ the vector boson mass, and $\alpha$ is a gauge parameter. $\mathcal{L}_{\mathrm{GF}}$ is the gauge fixing term defined by

$$
\mathcal{L}_{\mathrm{GF}} \equiv-\frac{1}{2 \alpha}\left(\partial_{\mu} A^{\mu}+\alpha m B\right)^{2} .
$$

We work with the Minkowski metric, $g_{\mu \nu}=$ $\operatorname{diag}(1,-1,-1,-1)$. We shall deal with a massless fermion assuming that the mass is neglected, which should be valid at high temperatures. Our Lagrangian is invariant under the gauge transformation except for the gauge fixing term, $\mathcal{L}_{\mathrm{GF}}$ :

$$
\begin{gathered}
\psi(x) \rightarrow e^{i g \Lambda(x)} \psi(x), \\
A_{\mu}(x) \rightarrow A_{\mu}(x)+\partial_{\mu} \Lambda(x), \\
B(x) \rightarrow B(x)+m \Lambda(x) .
\end{gathered}
$$

There is no interaction between the Stueckelberg field and the fermion field, and we chose the gauge fixing term so that the interaction term between the vector field and the Stueckelberg field vanishes. We can drop the Stueckelberg field as far as a correlation function is concerned, while it cannot be when the thermodynamic potential is considered, where it is important to take into account the correct degrees of freedom.

The propagator of the free massive vector boson is now given by

$$
D_{\mu \nu}(p)=\frac{-1}{p^{2}-m^{2}}\left(g_{\mu \nu}-\frac{p_{\mu} p_{\nu}}{p^{2}-m^{2} \alpha}(1-\alpha)\right) .
$$

In the $\alpha \rightarrow \infty$ limit, the propagator tends to

$$
D_{\mu \nu}(p) \rightarrow \frac{-1}{p^{2}-m^{2}}\left(g_{\mu \nu}-\frac{p_{\mu} p_{\nu}}{m^{2}}\right),
$$

which is the massive vector-boson propagator in the Proca formalism. ${ }^{2}$

\footnotetext{
${ }^{2}$ Here we note that the propagator in the Proca formalism does not vanish but rather approaches a constant value in the $p \rightarrow \infty$ limit, in contrast to that in the Stueckelberg formalism. This causes the nonrenormalizability and leads to bad behavior at high temperature $[8,18,19]$.
} 
The fermion propagator $G(p)$ in the imaginary time formalism $[15,16]$ is expressed with the self-energy $\Sigma(p)$ as

$$
G(p)=\frac{1}{\not p-\Sigma(p)},
$$

where $p^{0}=i \omega_{m}=i(2 m+1) \pi T$ is the Matsubara frequency for the fermion. Note that $G(p)$ and $\Sigma(p)$ are $4 \times 4$ matrices with the spinor indices. The retarded fermion propagator is given by an analytic continuation, $i \omega_{m} \rightarrow \omega+i \epsilon$ :

$$
G^{\mathrm{R}}(\mathbf{p}, \omega)=G(\mathbf{p}, \omega+i \epsilon)=\frac{1}{\omega \gamma^{0}-\mathbf{p} \cdot \gamma-\Sigma^{\mathrm{R}}(\mathbf{p}, \omega)},
$$

where the retarded self-energy is given by

$$
\Sigma^{\mathrm{R}}(\mathbf{p}, \omega)=\Sigma(\mathbf{p}, \omega+i \epsilon) .
$$

Introducing the projection operator on the (anti-)particle sector $\Lambda_{ \pm}(\mathbf{k})=\left(1 \pm \gamma^{0} \gamma \cdot \hat{\mathbf{k}}\right) / 2$, we can decompose the retarded propagator and self-energy into the respective sector as follows:

$$
G^{\mathrm{R}}(\mathbf{p}, \omega)=G_{+}(\mathbf{p}, \omega) \Lambda_{+}(\mathbf{p}) \gamma^{0}+G_{-}(\mathbf{p}, \omega) \Lambda_{-}(\mathbf{p}) \gamma^{0},
$$

$$
\Sigma^{\mathrm{R}}(\mathbf{p}, \omega)=\Sigma_{+}(\mathbf{p}, \omega) \Lambda_{+}(\mathbf{p}) \gamma^{0}+\Sigma_{-}(\mathbf{p}, \omega) \Lambda_{-}(\mathbf{p}) \gamma^{0},
$$

with $\Sigma_{ \pm}(\mathbf{p}, \omega)=\operatorname{Tr}\left(\Sigma^{\mathrm{R}}(\mathbf{p}, \omega) \Lambda_{ \pm}(\mathbf{p}) \gamma^{0}\right) / 2$.

In the particle sector, the pole $\omega_{\mathbf{p}}=\omega(\mathbf{p})$ satisfies the following equation:

$$
G_{+}^{-1}\left(\mathbf{p}, \omega_{\mathbf{p}}\right)=\omega_{\mathbf{p}}-|\mathbf{p}|-\Sigma_{+}\left(\mathbf{p}, \omega_{\mathbf{p}}\right)=0 .
$$

From the analyticity of the retarded propagator, the pole is located on the real axis or the lower half plane of complex $\omega$. If the imaginary part of the pole is small, the pole is well described in terms of a quasiparticle picture, where the real part of the pole corresponds to the energy, while the imaginary part to the decay width of the quasiparticle. If the imaginary part is large, then it would be meaningless to consider excitations in terms of any particle picture. It is known that the self-energy at zero momentum has the following symmetry:

$$
\begin{gathered}
\operatorname{Re} \Sigma_{+}(\mathbf{0},-\omega)=-\operatorname{Re} \Sigma_{+}(\mathbf{0}, \omega), \\
\operatorname{Im} \Sigma_{+}(\mathbf{0},-\omega)=\operatorname{Im} \Sigma_{+}(\mathbf{0}, \omega),
\end{gathered}
$$

which implies that if there exists a fermion pole at $z=$ $\omega-i \Gamma$ at zero momentum, there is also a pole at $-\omega-i \Gamma$ at zero momentum.

Once the self-energy $\Sigma_{+}(\mathbf{p}, \omega)$ is obtained, the spectral function of the (anti-)particle sector is expressed as

$$
\begin{aligned}
& \rho_{ \pm}(\mathbf{p}, \omega) \\
& =-\frac{1}{\pi} \operatorname{Im} G_{ \pm}(\mathbf{p}, \omega) \\
& =-\frac{1}{\pi} \frac{\operatorname{Im} \Sigma_{ \pm}(\mathbf{p}, \omega)}{\left(\omega \mp|\mathbf{p}|-\operatorname{Re} \Sigma_{ \pm}(\mathbf{p}, \omega)\right)^{2}+\operatorname{Im} \Sigma_{ \pm}^{2}(\mathbf{p}, \omega)}
\end{aligned}
$$

When the peak is narrow enough, the position of the peak is given by $\omega_{\mathbf{p}}=|\mathbf{p}|+\operatorname{Re} \Sigma_{+}\left(\mathbf{p}, \omega_{\mathbf{p}}\right)$, and the width of the peak is given by $-\operatorname{Im} \Sigma_{+}\left(\mathbf{p}, \omega_{\mathbf{p}}\right) /\left(2 \omega_{\mathbf{p}}\right)$.

\section{B. Calculation at one-loop order}

Now let us evaluate the self-energy $\Sigma(p)$ at one-loop order; the corresponding diagram is shown in Fig. 1. $\Sigma(p)$ is expressed as

$$
\begin{aligned}
\Sigma\left(\mathbf{p}, i \omega_{m}\right)= & -g^{2} T \sum_{n} \int \frac{d^{3} \mathbf{k}}{(2 \pi)^{3}} \gamma^{\mu} G_{0}\left(\mathbf{k}, i \omega_{n}\right) \gamma^{\nu} D_{\mu \nu} \\
& \times\left(\mathbf{p}-\mathbf{k}, i \omega_{m}-i \omega_{n}\right) \\
= & g^{2} T \sum_{n} \int \frac{d^{3} \mathbf{k}}{(2 \pi)^{3}} \gamma^{\mu} \frac{k}{k^{2}} \gamma^{\nu} \frac{1}{l^{2}-m^{2}} \\
& \times\left(g_{\mu \nu}-(1-\alpha) \frac{l_{\mu} l_{\nu}}{l^{2}-m^{2} \alpha}\right),
\end{aligned}
$$

where $G_{0}$ is the propagator of the free fermion, $l \equiv p-k$, and $k^{0}=i \omega_{n}=i(2 n+1) T$. Some manipulations lead to

$$
\begin{aligned}
\Sigma\left(\mathbf{p}, i \omega_{m}\right)= & -2 g^{2} \gamma^{\mu} \tilde{B}_{\mu}\left(\mathbf{p}, i \omega_{m} ; m\right) \\
& +\frac{g^{2}}{m^{2}}\left[p \left(p^{2}\left(\tilde{B}\left(\mathbf{p}, i \omega_{m} ; \sqrt{\alpha} m\right)-\tilde{B}\left(\mathbf{p}, i \omega_{m} ; m\right)\right)\right.\right. \\
& \left.-m^{2}\left(\alpha \tilde{B}\left(\mathbf{p}, i \omega_{m} ; \sqrt{\alpha} m\right)-\tilde{B}\left(\mathbf{p}, i \omega_{m} ; m\right)\right)\right) \\
& \left.-p^{2} \gamma^{\mu}\left(\tilde{B}_{\mu}\left(\mathbf{p}, i \omega_{m} ; \sqrt{\alpha} m\right)-\tilde{B}_{\mu}\left(\mathbf{p}, i \omega_{m} ; m\right)\right)\right] .
\end{aligned}
$$

The retarded self-energy $\Sigma^{\mathrm{R}}(\mathbf{p}, \omega)$ in the one-loop approximation is given by the analytic continuation $i \omega_{m} \rightarrow$ $\omega+i \epsilon$ from $\Sigma\left(\mathbf{p}, i \omega_{m}\right)$. Here, we have introduced the following loop functions:

$$
\tilde{B}\left(\mathbf{p}, i \omega_{m} ; m\right) \equiv T \sum_{n} \int \frac{d^{3} \mathbf{k}}{(2 \pi)^{3}} \frac{1}{(k-p)^{2}-m^{2}} \frac{1}{k^{2}},
$$

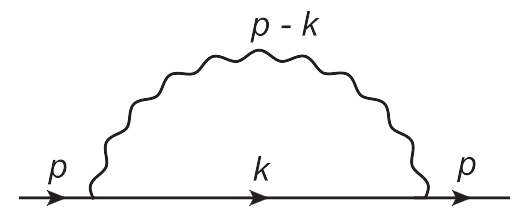

FIG. 1. The diagram which contributes to the fermion selfenergy at one-loop order. The solid line represents the fermion and the wavy line represents the massive vector boson. 
DAISUKE SATOW, YOSHIMASA HIDAKA, AND TEIJI KUNIHIRO

PHYSICAL REVIEW D 83, 045017 (2011)

$$
\tilde{B}^{\mu}\left(\mathbf{p}, i \omega_{m} ; m\right) \equiv T \sum_{n} \int \frac{d^{3} \mathbf{k}}{(2 \pi)^{3}} \frac{k^{\mu}}{(k-p)^{2}-m^{2}} \frac{1}{k^{2}} .
$$

We see that there are two kinds of mass in Eq. (2.18), $m$ and $\sqrt{\alpha} m$, the latter of which is unphysical because it depends on the gauge parameter. However, the existence of such an unphysical mass causes two different high temperature limits as will be shown in Sec. IV. We will also show that $\Sigma(p)$ approaches the fermion self-energy in QED if we take the massless limit $m \rightarrow 0$, which is not the case in the Proca formalism. It should be noted here that Eq. (2.18) shows that there is a special value of $\alpha$ : when $\alpha=1$, the terms containing the unphysical mass are all cancelled out and only the first term remains, i.e., $\Sigma\left(\mathbf{p}, i \omega_{m}\right)_{\alpha=1}=$ $-2 g^{2} \gamma_{\mu} \tilde{B}^{\mu}\left(\mathbf{p}, i \omega_{m} ; m\right)$.

The self-energy in the (anti-)particle sector in the one-loop approximation is given by $\Sigma_{ \pm}(\mathbf{p}, \omega)=$ $\operatorname{Tr}\left(\Sigma^{\mathrm{R}}(\mathbf{p}, \omega) \Lambda_{ \pm}(\mathbf{p}) \gamma^{0}\right) / 2$. Then, as is derived in Appendix B, we have for the imaginary part of $\Sigma_{+}(\mathbf{p}, \omega)$,

$$
\begin{aligned}
\operatorname{Im} \Sigma_{+}(\mathbf{p}, \omega)= & -\frac{g^{2}}{32 \pi|\mathbf{p}|^{2} m^{2}} \int_{E_{f}^{\prime+}}^{E_{f}^{\prime-}} d E_{f}\left(f\left(E_{f}\right)+n\left(E_{f}-\omega\right)\right)\left[\left(-p^{2}+m^{2} \alpha\right)(|\mathbf{p}|-\omega)^{2}+2 p^{2} E_{f}(\omega-|\mathbf{p}|)\right] \\
& +\frac{g^{2}}{32 \pi|\mathbf{p}|^{2} m^{2}} \theta\left(-p^{2}\right)\left[p^{2}(\omega-|\mathbf{p}|) \pi^{2} T^{2}+\omega(\omega-|\mathbf{p}|)\left(-\omega p^{2}-(\omega-|\mathbf{p}|)\left(-p^{2}+m^{2} \alpha\right)\right)\right] \\
& +\frac{g^{2}}{32 \pi|\mathbf{p}|^{2} m^{2}} \int_{E_{f}^{+}}^{E_{f}^{-}} d E_{f}\left(f\left(E_{f}\right)+n\left(E_{f}-\omega\right)\right)\left[\left(-p^{2}+m^{2}\right)\left((|\mathbf{p}|-\omega)^{2}-2 m^{2}\right)+2\left(p^{2}-2 m^{2}\right) E_{f}(\omega-|\mathbf{p}|)\right] \\
& -\frac{g^{2}}{32 \pi|\mathbf{p}|^{2} m^{2}} \theta\left(-p^{2}\right)\left[\left(p^{2}-2 m^{2}\right)(\omega-|\mathbf{p}|) \pi^{2} T^{2}+\omega\left[2 m^{4}-p^{2}\left(|\mathbf{p}|(\omega-|\mathbf{p}|)+m^{2}\right)\right]\right]
\end{aligned}
$$

where $p^{2}=\omega^{2}-|\mathbf{p}|^{2}, E_{f}^{ \pm}=\left(\omega^{2}-|\mathbf{p}|^{2}-m^{2}\right) /(2(\omega \pm$ $|\mathbf{p}|))$ and $E_{f}^{\prime \pm}=\left(\omega^{2}-|\mathbf{p}|^{2}-\alpha m^{2}\right) /(2(\omega \pm|\mathbf{p}|))$.

The real part $\operatorname{Re} \Sigma_{+}(\mathbf{p}, \omega)$ may be obtained using the dispersion relation from the imaginary part. Especially, the finite temperature part of the real part of the self-energy, $\Sigma_{+}(\mathbf{p}, \omega)_{T \neq 0} \equiv \Sigma_{+}(\mathbf{p}, \omega)-\Sigma_{+}(\mathbf{p}, \omega)_{T=0}$, is expressed as

$$
\operatorname{Re} \Sigma_{+}(\mathbf{p}, \omega)_{T \neq 0}=-\frac{1}{\pi} \mathrm{P} \int_{-\infty}^{\infty} d \omega^{\prime} \frac{\operatorname{Im} \Sigma_{+}\left(\mathbf{p}, \omega^{\prime}\right)_{T \neq 0}}{\omega-\omega^{\prime}} .
$$

Here $\mathrm{P}$ denotes the principal value. The zero temperature part of $\operatorname{Re} \Sigma_{+}(\mathbf{p}, \omega)$ is not determined by Eq. (2.22) because it has ultraviolet divergence. We make renormalization using twice-subtracted dispersion relation, which reads

$$
\begin{aligned}
& \operatorname{Re} \Sigma_{+}(\mathbf{p}, \omega)_{T=0}=c_{0}+c_{1}(\omega-|\mathbf{p}|) \\
& \quad+\frac{(\omega-|\mathbf{p}|)^{2}}{\pi} \mathrm{P} \int_{-\infty}^{\infty} d z \frac{\operatorname{Im} \Sigma_{+}(\mathbf{p}, z)_{T=0}}{(z-|\mathbf{p}|)^{2}(z-\omega)} .
\end{aligned}
$$

We impose the on-shell renormalization condition, $\Sigma_{+}(\mathbf{p}, \omega=|\mathbf{p}|)=0$ and $\partial \Sigma_{+}(\mathbf{p}, \omega) /\left.\partial \omega\right|_{\omega=|\mathbf{p}|}=0$, to determine $c_{0}$ and $c_{1}$. The vacuum part of $\operatorname{Im} \Sigma_{+}(\mathbf{p}, \omega)$ is obtained by taking the $T \rightarrow 0$ limit of Eq. (2.21);

$$
\begin{aligned}
\operatorname{Im} \Sigma_{+}(\mathbf{p}, \omega)_{T=0}= & \frac{g^{2}}{32 \pi m^{2}} \frac{\operatorname{sgn}(\omega)}{p^{2}}(\omega-|\mathbf{p}|) \\
& \times\left[\theta\left(p^{2}-\alpha m^{2}\right)\left(p^{2}-\alpha m^{2}\right)^{2}\right. \\
& \left.-\theta\left(p^{2}-m^{2}\right) \frac{\left(p^{2}+2 m^{2}\right)\left(p^{2}-m^{2}\right)^{2}}{p^{2}}\right] .
\end{aligned}
$$

Thus, we arrive at

$$
\begin{gathered}
\operatorname{Re} \Sigma_{+}(\mathbf{p}, \omega)_{T=0}=\frac{g^{2} p^{2}}{32 \pi^{2} m^{2}}(\omega-|\mathbf{p}|)\left[\frac{2 m^{4}}{p^{4}}-(2+\alpha) \frac{m^{2}}{p^{2}}\right. \\
+\ln \left|\frac{p^{2}-m^{2}}{p^{2}-m^{2} \alpha}\right|+\alpha \frac{m^{2}}{p^{2}}\left(-2+\frac{m^{2} \alpha}{p^{2}}\right) \ln \left|\frac{\alpha\left(p^{2}-m^{2}\right)}{p^{2}-m^{2} \alpha}\right| \\
\left.+\frac{m^{2}}{p^{2}}\left(-2 \alpha+\left(3+\alpha^{2}\right) \frac{m^{2}}{p^{2}}-2 \frac{m^{4}}{p^{4}}\right) \ln \left|\frac{m^{2}}{p^{2}-m^{2}}\right|\right]
\end{gathered}
$$

As mentioned before, our theory based on the Stueckelberg formalism approaches QED at high enough temperature where the masses are negligible in comparison with $T$. Let us see this. For $T \gg g T \gg m, \sqrt{\alpha} m$, the imaginary and real part of the self-energy are reduced to

$$
\begin{aligned}
\operatorname{Im} \Sigma_{+}(\mathbf{p}, \omega)_{T \rightarrow \infty} \simeq & \frac{g^{2}}{32 \pi|\mathbf{p}|^{2} m^{2}} \theta\left(-p^{2}\right)\left[p^{2}(\omega-|\mathbf{p}|) \pi^{2} T^{2}\right. \\
& \left.-\left(p^{2}-2 m^{2}\right)(\omega-|\mathbf{p}|) \pi^{2} T^{2}\right] \\
= & \frac{g^{2} \theta\left(-p^{2}\right)}{16|\mathbf{p}|^{2}} \pi T^{2}(\omega-|\mathbf{p}|)
\end{aligned}
$$

$\operatorname{Re} \Sigma_{+}(\mathbf{p}, \omega)_{T \rightarrow \infty} \simeq \frac{g^{2} T^{2}}{16|\mathbf{p}|^{2}}\left(2|\mathbf{p}|+(|\mathbf{p}|-\omega) \ln \left|\frac{\omega+|\mathbf{p}|}{\omega-|\mathbf{p}|}\right|\right)$,

respectively. Here, we have retained only the terms that are proportional to $T^{2}$ in Eq. (2.21). These equations, (2.26) and (2.27), coincide exactly with the well-known results in the HTL approximation in QED [1-4]. There is a caveat in 
the above manipulation, which has been taken for granted in the usual derivation of the HTL approximation in the gauge theory: The ignored terms may become comparable to terms that are proportional to $T^{2}$ in some gauges and hence the above naive power counting turns out to be invalid. We will analyze this possibility in Sec. IV.

\section{NUMERICAL RESULTS}

In this section, we show the numerical results of the fermion spectral function and the fermion poles at various temperatures. In the following, the coupling constant is fixed to a small value, $g=0.5$, so that the analysis based on the one-loop calculation can be valid: Except when the coupling constant dependence of the pole is analyzed, the coupling constant will be fixed to $g=0.5$. On the other

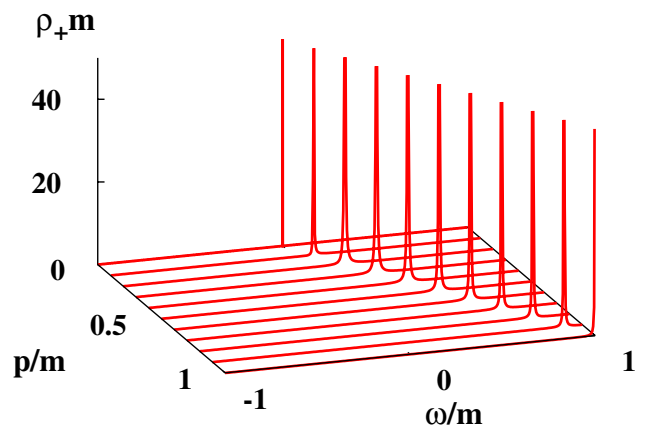

FIG. 2 (color online). The fermion spectral function in the particle sector $\rho_{+}$as a function of energy $\omega$ and momentum $p$ for $T=0.4 m, g=0.5, \alpha=1$.

$\mathbf{T} / \mathbf{m}=\mathbf{1 . 0}$

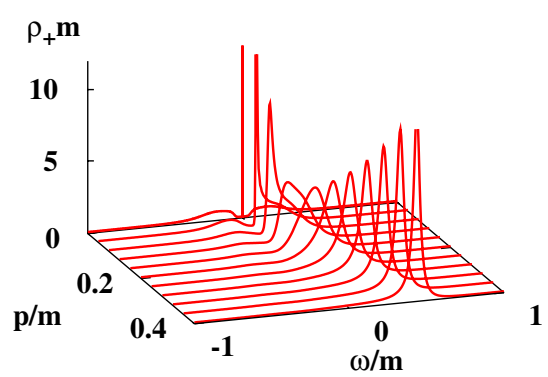

$\mathbf{T} / \mathbf{m}=\mathbf{2 . 0}$

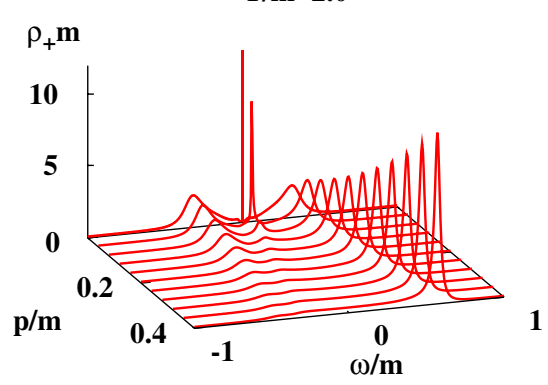

hand, the gauge parameter, $\alpha$, will be varied freely in order to see the gauge dependence of the spectral properties of the fermion calculated at the one-loop level.

\section{A. Low temperature $(T \ll m)$}

In this subsection, we show numerical results at a temperature so low that the $T$ dependence of the results is hardly seen, which may check the validity of our analytical and numerical calculations.

Figure 2 shows the fermion spectral function in the particle sector (with a positive particle number) at $T=$ $0.4 m$ for $\alpha=1$. A very narrow peak appears near $\omega=|\mathbf{p}|$, which is very reminiscent of the zero temperature case. This is natural for $T \ll m$, because the thermal effect is exponentially suppressed by the Boltzmann factor $\sim \exp (-m / T)$, and hence the breaking of Lorentz symmetry is small. This small breaking of Lorentz symmetry implies that the particle pole is almost an on-shell value at $T=0$, i.e., $\omega=|\mathbf{p}|$, and hence the gauge dependence of the pole hardly appears.

\section{B. Intermediate temperature $(T \sim m)$}

We plot the spectral function of the particle sector at $T / m=1.0,1.5,2.0,2.5$ for $\alpha=1$ in Fig. 3. We can see that the spectral function at these temperatures have structures qualitatively different from that at low temperature: Even at $T / m=1.0$, we see a split of a peak around the origin, seen in Fig. 2 into two peaks with a small bump in the negative energy region, which is reminiscent of the antiplasmino peak known in QED/QCD at high $T$. These

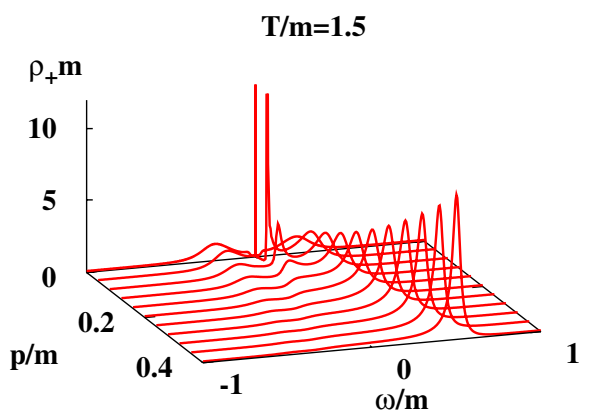

$\mathrm{T} / \mathrm{m}=\mathbf{2 . 5}$

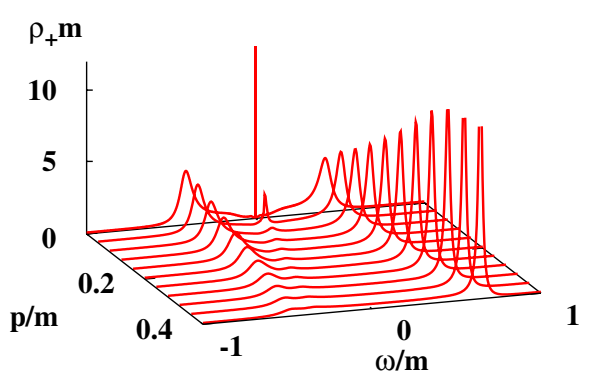

FIG. 3 (color online). The fermion spectral function in the particle sector $\rho_{+}$as a function of energy $\omega$ and momentum $p$ at $g=0.5$, $\alpha=1$ at various temperatures. 


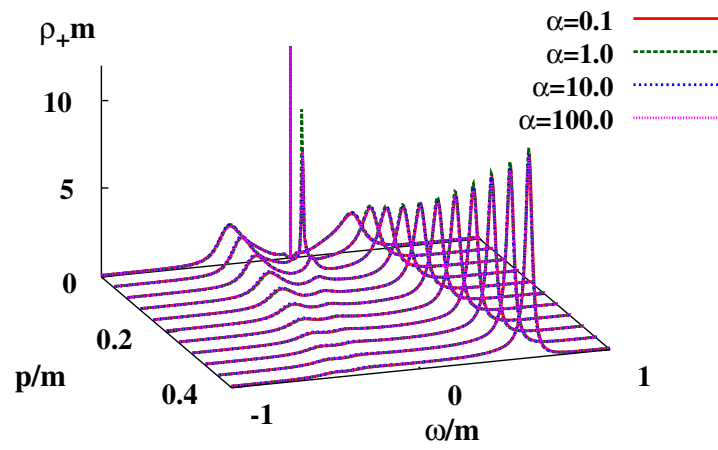

FIG. 4 (color online). The spectral fermion function in the particle sector $\rho_{+}$as a function of energy $\omega$ and momentum $p$ at $T=2.0 \mathrm{~m}, \mathrm{~g}=0.5$.

features are enhanced as $T$ is raised, and we see a clear three-peak structure around $|\mathbf{p}|=0$ at $T / m=2.0$ with a prominent peak and a clear bump in the positive and negative energy region, respectively. We also see that the peak near the origin is attenuated as $T$ is further raised up to $2.5 \mathrm{~m}$.

Since it is known that the details of the shape of the spectral function may be gauge dependent in general, let us see how the three-peak structure depends on the gauge parameter. Figure 4 shows the gauge-parameter dependence of the fermion spectral function in the particle sector at $T=2.0 \mathrm{~m}$; the gauge parameter is varied as $\alpha=0.1,1$, 10 , and 100. One might find only a single curve of the spectral function in the figure, although this figure actually shows four curves of it with different $\alpha$; thus, it clearly tells us that the shape of the spectral function at $T=2 \mathrm{~m}$ with a three-peak structure is virtually independent of the gauge parameter.

The virtual gauge independence of the shape of the spectral function implies that the pole of the propagator is also the case. We show the gauge (in)dependence of the pole in the positive energy region at $|\mathbf{p}|=0$ with a particle number in the left panel of Fig. 5, which shows that the pole position is almost independent of the choice of the gauge parameter, as anticipated: Note that the gauge parameter is varied in a wider range than in Fig. 4, i.e., $\alpha=0.01,0.1,1,10,100,1000$. A remark is in order here: The pole in the negative energy region at $|\mathbf{p}|=0$ has the same properties as that in the positive energy region, as is assured by Eqs. (2.14) and (2.15).

Such a gauge independence of the poles necessarily reflects in that of the spectral function. The right panel of Fig. 5 shows the fermion spectral function at zero momentum for the wide range of $\alpha$ up to 1000, together with that obtained in the Proca formalism. ${ }^{3}$ From this figure, we confirm that the spectral function at zero momentum is virtually gauge independent for the wide range of $\alpha$.

\footnotetext{
${ }^{3}$ The rapid decrease of the spectral function in $|\omega| \leq 0.1$ is caused by the exponential damping of $\operatorname{Im} \Sigma_{+}(\mathbf{0}, \omega)$ in that region.
}

We also note that the position and the width of the peaks coincide with the real and imaginary part of the poles, respectively, which is due to the fact that the imaginary part of the poles is small in comparison with the real part, as seen in the left panel of Fig. 5. Thus, the shape of the spectral function with a three-peak structure necessarily gets to have almost no gauge dependence.

We show the coupling constant dependence of the fermion pole at zero momentum in Fig. 6 for $T / m=2.0$. The real part is almost proportional to $g$, like that in QED in the HTL approximation. The coupling constant dependence of the imaginary part is not large.

What is the mechanism for realizing the three-peak structure of the fermion spectral function? Figure 7 shows the real and imaginary part of the self-energy for $T / \mathrm{m}=$ 2.0 and $\alpha=1.0$ at $|\mathbf{p}|=0$, together with the corresponding spectral function. A detailed analysis of the imaginary part tells us that the peaks of the imaginary part correspond to a Landau damping of the fermion by a scattering with thermally excited bosons. Since these features of the fermion self-energy are very similar to those shown in [8], the mechanism for realizing the three-peak structure found in our formalism is understood to be the same as discussed in [8].

\section{High temperature $(T \gg m)$}

In this subsection, we show numerical results in the high temperature $(T \gg m)$ region, where the mass of the vector boson (and the fermion) can be neglected in comparison with $T$, i.e., $m / T \rightarrow 0$; this means that $T$ itself may not be infinitely large.

We show the fermion pole in the positive energy region in the left panel of Fig. 8 at $T=40.0 \mathrm{~m}$ and for $\alpha=0.1,1$, 10 and 100. The pole in the Proca formalism and that in the HTL approximation in QED are also shown. We see that the gauge dependence of the fermion pole is no longer negligible. Since the exact pole position in the complex energy plane should be gauge independent [12], the above result suggests that the one-loop analysis is no longer valid in this high- $T$ region in contrast to the $T \ll m$ and $T \sim m$ regions, at least in some gauge fixing. We will present a detailed discussion on how the gauge dependence arises at the high $T$ region in Sec. IV.

One should notice that the pole for $\alpha=0.1$ is located in the upper energy plane, which could be problematic because it implies a loss of the analyticity of the retarded propagator and also the negativeness of the spectral function, as seen from Eq. (2.16).

For $\alpha \sim 1$, there appear clear two peaks in the spectral function in a robust way, as shown in Fig. 9; the two peaks are found to tend to the normal fermion(particle) and the antiplasmino of QED in the HTL approximation [1-4], respectively; see Sec. IV.

The other peak persists at the origin in the energymomentum space. One can confirm that its residue is of 

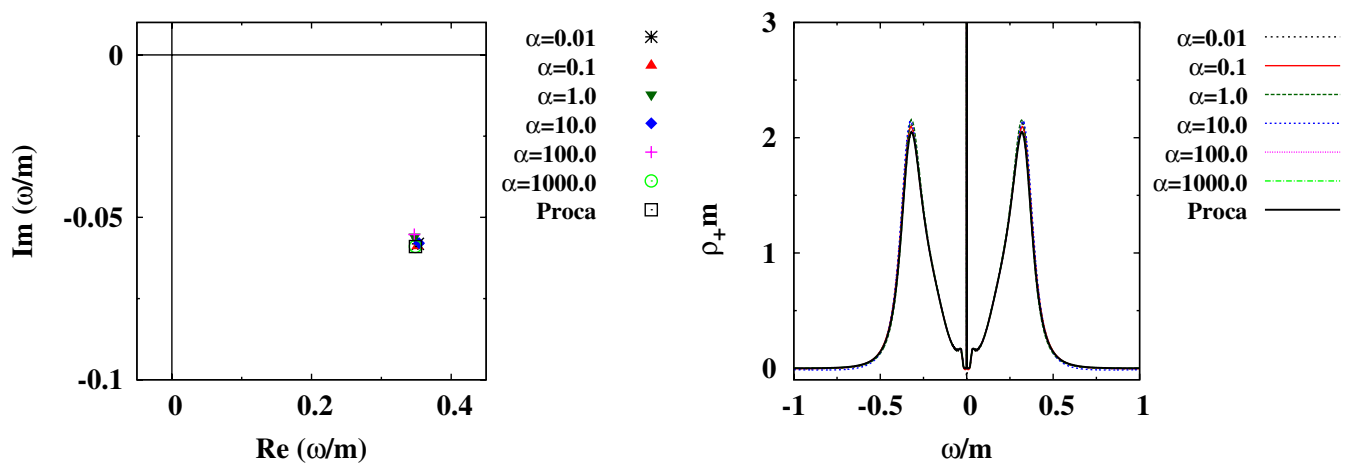

FIG. 5 (color online). The left panel shows the gauge dependence of the fermion pole with a positive particle number in the positive energy region for $T=2.0 \mathrm{~m}, \mathrm{~g}=0.5$ at zero momentum: the horizontal and vertical axes denote the real and imaginary part of the energy, respectively. The right panel shows the fermion spectral function in the particle sector $\rho_{+}$as a function of energy $\omega$ for the same $T, g$ as in the left panel.
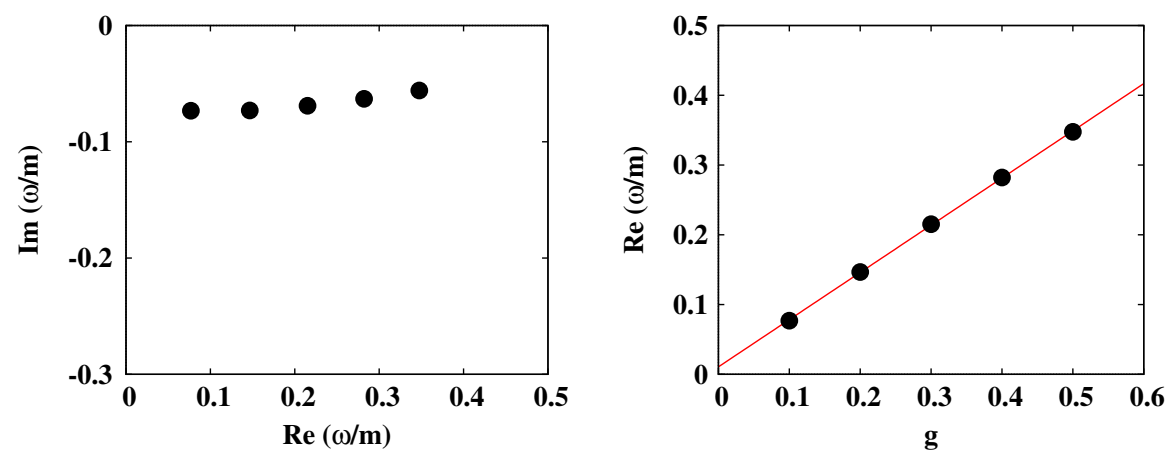

FIG. 6 (color online). The left panel shows the coupling constant dependence of the fermion pole with a particle number in the positive energy region for $T / m=2.0$ and $\alpha=1.0$, at zero momentum: the horizontal and the vertical axis denote the real and imaginary part of the energy, respectively. The dots from left to right correspond to $g=0.1,0.2,0.3,0.4,0.5$, respectively. The right panel shows the coupling constant dependence of the real part of the fermion(particle) pole in the positive energy region at zero momentum for the same $T / m$ and $\alpha$. The solid line is the fitted linear function.

the order of $m^{4} /\left(g^{2} T^{4}\right)$, which is very small if we consider the $g T \gg m$ case, by making power counting. Such a peak at the origin was also obtained in [8], though in the Proca formalism. One should also remark that such a peak at the zero energy is not obtained in QED in the HTL approximation, in which the vector boson mass is set to zero from the beginning, in contrast to the present case. ${ }^{4}$ It should be intriguing to explore whether this peak at the origin extends to a finite- $|\mathbf{p}|$ region, and hence the three-peak structure of the fermion spectral function persists even in such a high- $T$ region, i.e., for $T \gg m$. In fact, this is a challenging problem in quantum field theory at finite temperature, because a sensible analysis of such an infrared region requires a systematic method to remove the socalled pinch singularities [20]. This task is beyond the

\footnotetext{
${ }^{4}$ The absence of a peak at the vanishing energy in QED with the HTL approximation is easily understood as follows: In the HTL approximation of QED, $\operatorname{Re} \Sigma_{+}(\mathbf{0}, \omega)$ behaves as $1 / \omega$ in the $\omega \rightarrow 0$ limit. Thus, at $\omega=0$, the pole condition Eq. (2.13) will not be satisfied and hence a pole at the origin cannot exist.
}

scope of the present work, and we leave such an analysis to a future work [21].

Our numerical calculation has shown that one can have virtually gauge-independent results even in the one-loop analysis if the gauge parameter is in the region $\alpha \sim 1$. We shall argue in Sec. IV that the perturbative expansion should be valid for $\alpha \ll 1 / g$. It means that the spectral function of a fermion coupled with a massive vector boson as calculated in the Stueckelberg formalism nicely approaches that in QED in the HTL approximation at high $T$ irrespective of the choice of the gauge parameter $\alpha$, if the order of $\alpha$ is confined to $\alpha \ll 1 / g$. This is actually already suggested by the asymptotic form Eq. (2.27) for $T \gg m, \sqrt{\alpha} m$.

\section{ANALYSIS OF GAUGE DEPENDENCE OF THE POLE AT HIGH TEMPERATURE $(T \gg m)$}

The pole position of the fermion propagator is virtually independent of the gauge parameter $\alpha$ for the cases of $T \ll m$ and $T \sim m$ : The former case is simply because the thermal contribution due to a boson with a mass $m$ is 
DAISUKE SATOW, YOSHIMASA HIDAKA, AND TEIJI KUNIHIRO

PHYSICAL REVIEW D 83, 045017 (2011)

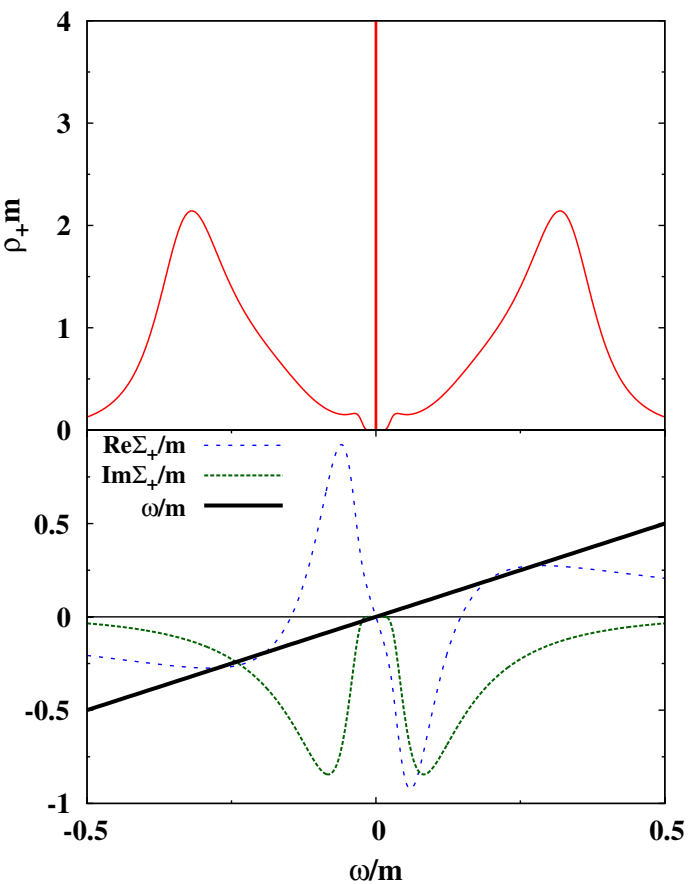

FIG. 7 (color online). The upper figure shows the fermion spectral function in the particle sector $\rho_{+}$as a function of energy $\omega$ for $g=0.5, T=2.0 m$, and $\alpha=1$. The lower figure shows the real part and the imaginary part of the fermion self-energy as functions of energy $\omega$ for the same $g, T$, and $\alpha$. The dotted line denotes $\omega$. The intersection points between this line and $\operatorname{Re} \Sigma_{+}(\mathbf{0}, \omega)$ are the solutions of the dispersion relation.

greatly suppressed by a Boltzmann factor $\exp (-m / T) \ll$ 1 when $T \ll m$. By contrast, for $T \gg m$, the numerical results in Sec. IIIC show that the pole of the fermion propagator has a large gauge dependence for large $\alpha$. In this section, we discuss the gauge dependence of the pole of the fermion propagator at weak coupling at high temperature. In particular, we focus on the region $g T \gg m$. In this region, one expects that the mass of the vector boson can be neglected, and thus the self-energy approach that in the HTL approximation of QED [1-4], in which the fermion has the pole of order $g T$. Therefore, we analyze the pole of the fermion propagator by assuming $\omega \sim g T$. Here we introduce a small dimensionless parameter,

$$
\lambda \equiv\left(\frac{m}{g T}\right)^{2} \ll 1 .
$$

Thus, we have two small dimensionless parameters, $g$ and $\lambda$, which are treated as independent parameters, so that the self-energy is expanded by combined powers of $g$ and $\lambda$. If the power of $g$ and $\lambda$ are both positive, the high temperature limit will be well defined and smoothly connected to that of QED. However, as will be shown below, an inverse power of $\lambda$ appears at the one-loop level when the gauge parameter is large, and hence the high temperature limit becomes inevitably different from that of QED.

In the following analysis, we put $|\mathbf{p}|=0$ for simplicity. The pole position obtained in the perturbation theory generally depends on the gauge parameter as well as $g, \lambda$, and $T$ due to the truncation of the perturbative expansion. We parametrize the pole $\omega_{\text {pole }}$ of the fermion propagator as

$$
\omega_{\text {pole }}=g T F(g, \lambda, \alpha),
$$

where $F(g, \lambda, \alpha)$ is a function of order one, and depends on the gauge parameter $\alpha$. If the limit,

$$
F_{0} \equiv \lim _{g \rightarrow 0} F(g, \lambda, \alpha)
$$

is independent of $\alpha$, then the pole is independent of the gauge parameter at the order $g T$. Thus, one sees that the gauge-dependent part may be defined by

$$
\delta \omega_{\text {pole }}(g, \lambda, \alpha) \equiv \omega_{\text {pole }}(g, \lambda, \alpha)-\omega_{\text {pole }}^{0}(\lambda),
$$

where $\omega_{\text {pole }}^{0}(\lambda) \equiv g T F_{0}(\lambda)$. As a reference, we recall that $F_{0}(\lambda=0)=1 /(2 \sqrt{2})$ in the case of QED [4]. When the inequality

$$
\omega_{\text {pole }}^{0}(\lambda) \gg \delta \omega_{\text {pole }}(g, \lambda, \alpha)
$$

is satisfied, the gauge dependence can be neglected. In reality with a finite $g$, the region of the gauge parameter
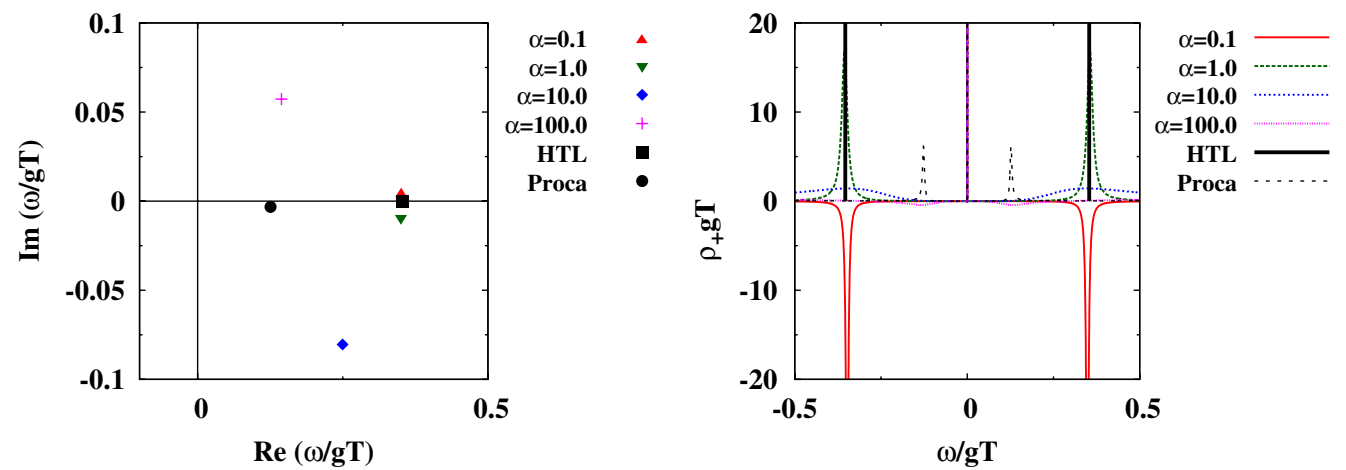

FIG. 8 (color online). The left panel shows the gauge dependence of the fermion pole at $p=0$ in the positive energy region at $T=40.0 \mathrm{~m}$ for $g=0.5$ : The horizontal and the vertical axis denote the real and imaginary part of energy. The right panel shows the fermion spectral function in the particle sector $\rho_{+}$as a function of energy $\omega$ for the same $T$ and $g$. 


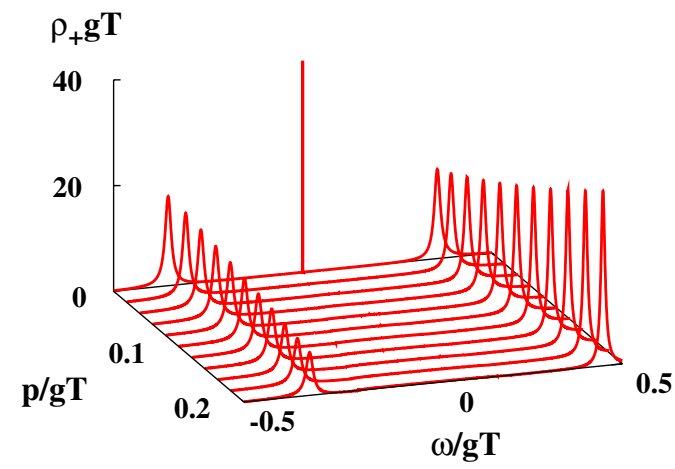

FIG. 9 (color online). The fermion spectral function in the particle sector $\rho_{+}$as a function of energy $\omega$ and momentum $p$ at $T=40.0 m$ with $g=0.5$ and $\alpha=1$.

satisfying Eq. (4.5) will be limited. We shall call the region that the gauge parameter satisfies Eq. (4.5) an adequate gauge-parameter region. The purpose of this section is to find the adequate gauge-parameter region.

Let us first show a numerical result of the real and the imaginary part of the pole at $T=40.0 \mathrm{~m}$ as functions of $\alpha$ in Fig. 10: For a large $\alpha(1 \ll \alpha)$, the $\alpha$ dependence of the real part of the pole is large, and especially for very large $\alpha$, say $\alpha \sim 3 \times 10^{4}$, the magnitude of it is no longer of $O(g T)$, but is of a smaller order, $O(m)$, as will be shown later. The imaginary part of the pole for $\alpha \ll 1$ is positive and apparently problematic because it means that the analyticity of the retarded propagator is lost and the fermion spectral function will become negative. As we shall show later, however, the absolute value of the imaginary part is of $O\left(g^{2} T\right)$ and should be considered together with higher order contributions. So the negative imaginary part with a small absolute value can be ignored in this order of the coupling.

Now we shall show that such an order estimate of the pole can be done analytically. We start with an analysis of the self-energy, under the condition that $\omega \sim g T$, by decomposing the self-energy (2.18) to seven parts,

$$
\Sigma_{+}(\mathbf{0}, \omega) \equiv \omega C(\omega)=\omega \sum_{n=1}^{7} C_{n}(\omega),
$$

where we have introduced the following dimensionless functions:

$$
\begin{gathered}
C_{1}(\omega)=-\frac{2 g^{2}}{\omega} \tilde{B} 0(\mathbf{0}, \omega ; m), \\
C_{2}(\omega)=+\frac{g^{2} \omega^{2}}{m^{2}} \tilde{B}(\mathbf{0}, \omega ; \sqrt{\alpha} m), \\
C_{3}(\omega)=-\frac{g^{2} \omega^{2}}{m^{2}} \tilde{B}(\mathbf{0}, \omega ; m), \\
C_{4}(\omega)=-g^{2} \alpha \tilde{B}(\mathbf{0}, \omega ; \sqrt{\alpha} m),
\end{gathered}
$$

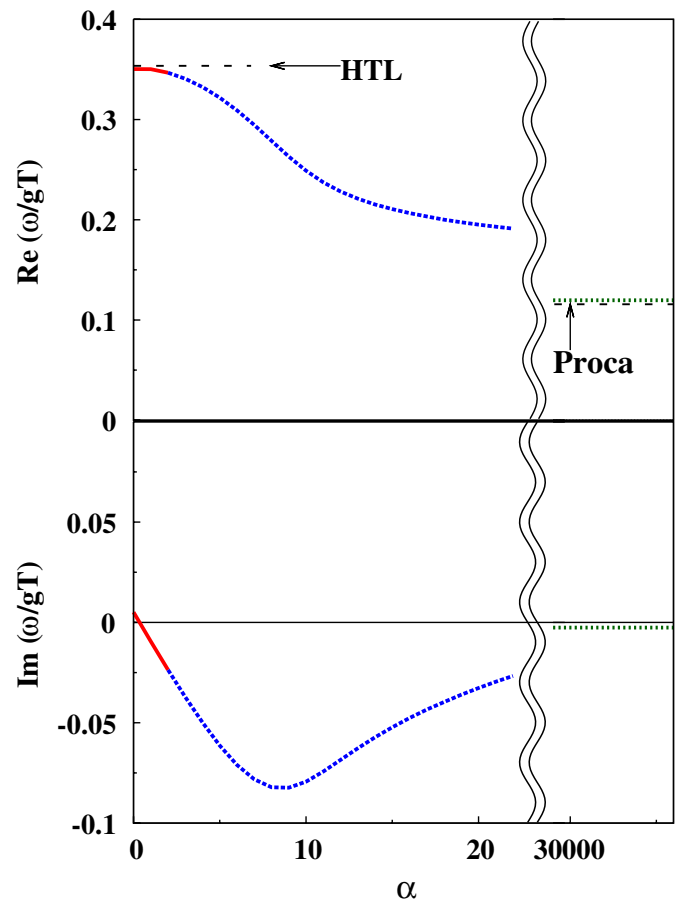

FIG. 10 (color online). The upper panel shows the gauge dependence of the real part of the pole in the positive energy region at zero momentum at $T=40.0 \mathrm{~m}$, for $g=0.5$. The horizontal and the vertical axes denote the gauge parameter $\alpha$ and the real part of the pole, respectively. The data whose $\alpha$ is smaller than the upper limit of adequate gauge parameter, $1 / \mathrm{g}=$ 2.0, are denoted by the solid line and the data whose $\alpha$ is larger than $1 / g$ by the dotted line. The data which satisfy $\alpha>$ $1 /\left(g^{2} \lambda\right)=1600$, which are expected to approach the value in the Proca formalism, are plotted with another dotted line. The upper and lower dashed lines show the thermal mass in the HTL approximation $g T /(2 \sqrt{2})$ and that in the Proca formalism $\sqrt{6} \mathrm{~m} / \sqrt{1+48 \lambda} \simeq 2.31 \mathrm{~m}$, respectively. The lower panel shows the gauge dependence of the imaginary part of the pole for the same $T$ and $g$ as in the upper panel.

$$
C_{5}(\omega)=+g^{2} \tilde{B}(\mathbf{0}, \omega ; m)
$$

$$
C_{6}(\omega)=-\frac{g^{2} \omega}{m^{2}} \tilde{B}^{0}(\mathbf{0}, \omega ; \sqrt{\alpha} m)
$$

$$
C_{7}(\omega)=+\frac{g^{2} \omega}{m^{2}} \tilde{B}^{0}(\mathbf{0}, \omega ; m) .
$$

Here $\tilde{B}(\mathbf{0}, \omega ; m)$ and $\tilde{B}^{0}(\mathbf{0}, \omega ; m)$ are obtained by performing the analytic continuation $\left(i \omega_{m} \rightarrow \omega+i \epsilon\right)$ to $\tilde{B}\left(\mathbf{0}, i \omega_{m} ; m\right)$ and $\tilde{B}^{0}\left(\mathbf{0}, i \omega_{m} ; m\right)$. From Eq. (2.13), one sees that the poles satisfy the condition

$$
C\left(\omega_{\text {pole }}\right)=1
$$

We first note that if the following equation has a root,

$$
\lim _{g \rightarrow 0} C(g T F)=1,
$$


there is a pole $\omega_{\text {pole }}=g T F$ of order $g T$. Furthermore, if the $F$ happens to be independent of $\alpha$, the pole is gauge independent. Therefore, let us take the following function as a measure of the gauge dependence, instead of Eq. (4.4):

$$
\delta C(g, \lambda, \alpha) \equiv C\left(\omega_{\text {pole }}^{0}\right)-1
$$

Then a criterion for the adequate gauge-parameter region may be given by

$$
\delta C(g, \lambda, \alpha) \ll 1 .
$$

We now make an order estimate of the seven terms defined in Eqs. (4.7), (4.8), (4.9), (4.10), (4.11), (4.12), and (4.13). One finds that this task is reduced to that of $\tilde{B}(\mathbf{0}, \omega ; m)$ and $\tilde{B}^{0}(\mathbf{0}, \omega ; m)$. The following relations are shown in Appendix $C$ :

(i) When $T \gg \omega \gg M$,

$$
\tilde{B}(\mathbf{0}, \omega ; M) \sim \frac{T}{\omega}, \quad \frac{\tilde{B}^{0}(\mathbf{0}, \omega ; M)}{\omega} \sim \frac{T^{2}}{\omega^{2}} .
$$

(ii) When $T \gg M \gg \omega$,

$$
\begin{gathered}
\tilde{B}(\mathbf{0}, \omega ; M) \sim\left\{\begin{array}{ll}
\frac{T}{\omega} & \text { for } M^{2} \ll \omega T \\
\frac{T^{2}}{M^{2}} & \text { for } M^{2} \gg \omega T
\end{array},\right. \\
\frac{\tilde{B}^{0}(\mathbf{0}, \omega ; M)}{\omega} \sim\left\{\begin{array}{ll}
\frac{T^{2}}{\omega^{2}} & \text { for } M^{2} \ll \omega T \\
\frac{T^{4}}{M^{4}} & \text { for } M^{2} \gg \omega T
\end{array} .\right.
\end{gathered}
$$

(iii) When $M \gg T \gg \omega$,

$$
\tilde{B}(\mathbf{0}, \omega ; M) \sim \frac{T^{2}}{M^{2}}, \quad \frac{\tilde{B}^{0}(\mathbf{0}, \omega ; M)}{\omega} \sim \frac{T^{4}}{M^{4}} .
$$

Here, $M$ denotes $m$ or $\sqrt{\alpha} m$, and the vacuum parts have been dropped. Using these relations, we will find the adequate gauge-parameter region in the following subsections, and the result is summarized in Fig. 11, which shows that the adequate gauge parameter is $\alpha \ll 1 / g$.

$C_{1}(\omega), C_{3}(\omega), C_{5}(\omega)$, and $C_{7}(\omega)$, which do not depend on the gauge parameter, are estimated to be

$$
\begin{aligned}
& C_{1}(\omega) \simeq g^{2} \frac{T^{2}}{8 \omega^{2}} \sim 1, \quad C_{3}(\omega) \sim g\left(\frac{1}{\lambda}\right), \\
& C_{5}(\omega) \sim g, \quad C_{7}(\omega) \sim\left(\frac{1}{\lambda}\right) .
\end{aligned}
$$

We remark that $C_{1}(\omega)$ coincides with the fermion selfenergy in the HTL approximation in QED, as it should.

We note that $C_{3}(\omega)$ and $C_{7}(\omega)$ are of the order of an inverse power of $\lambda$, which would make it impossible to take the massless limit. These "dangerous" terms are found to be nicely canceled out with other $C_{i}(\omega)$ 's when $\alpha$ is not so large, whereas for large $\alpha$, the cancellation does

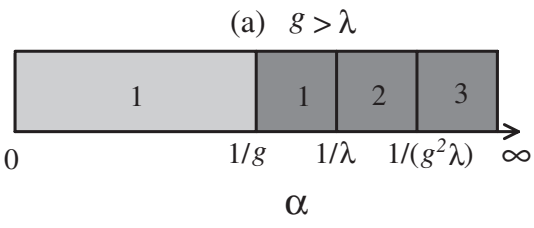

(b) $g<\lambda$

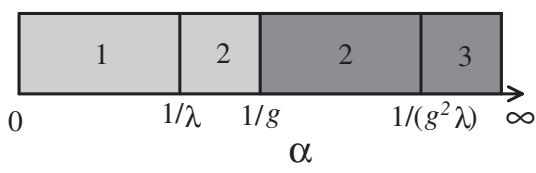

FIG. 11. The classification of the gauge-parameter regions depending on the relative magnitude of $\lambda=m^{2} /(g T)^{2}$ to $g$ at high temperature, i.e., for $g T \gg m$ or $\lambda \ll 1$. Each number in the figures corresponds to the case with same number in the text. The light gray regions are the adequate gauge-parameter regions for which Eq. (4.5) is satisfied, whereas the dark gray region are not.

not happen, and the condition $\delta C \ll 1$ cannot be satisfied, as will be shown below.

\section{A. $1 \gg \alpha \lambda$ (case 1)}

When $1 \gg \alpha \lambda$, the mass scale $m$ and $\sqrt{\alpha} m$ are negligible in comparison with $g T$, and then the self-energy coincides with that in the HTL approximation in QED in this approximation, because the nonleading terms can be neglected as we have seen in Sec. II.

Due to the order estimate in Eq. (4.18), we obtain

$$
C_{2}(\omega) \sim g\left(\frac{1}{\lambda}\right), \quad C_{4}(\omega) \sim g \alpha, \quad C_{6}(\omega) \sim\left(\frac{1}{\lambda}\right) .
$$

The leading terms of $C_{2}(\omega)$ and $C_{6}(\omega)$ cancel out ${ }^{5}$ with $C_{3}(\omega)$ and $C_{7}(\omega)$, respectively, and the terms of the order $g \alpha$ and $g$ remain. ${ }^{6}$ Thus, one sees that the gauge-dependent part is of the order of $g \alpha$, which means that the pole is gauge independent in practice, which also can be confirmed from Fig. 10, provided that the inequality $\alpha \ll 1 / g$ is satisfied; in this case, the adequate gauge-parameter region is the region of $\alpha$ which satisfies the above inequality.

Let us see that the imaginary part of the self-energy is of order $g^{2} T$ in the Landau gauge $(\alpha=0)$. For $\alpha=0$, the imaginary part of the self-energy is evaluated to be

\footnotetext{
${ }^{5}$ As seen from Eqs. (C14) and $(\mathrm{C} 19), \tilde{B}(p ; m)$ and $\tilde{B}^{0}(p ; m)$ yield terms that are proportional to $m$, so $C_{2}(\omega), C_{3}(\omega), C_{6}(\omega)$, and $C_{7}(\omega)$ seem to yield terms that are proportional to $m^{-1}$, and the massless limit $(m / T \rightarrow 0)$ cannot be taken. Actually, from Eqs. (C14) and (C19), we see that the terms discussed above cancel out. Therefore, we can take the massless limit, and the fermion self-energy approaches the one in QED.

${ }^{6}$ These terms come from the imaginary part of $\tilde{B}(\mathbf{0}, \omega ; m)$, $\tilde{B}^{0}(\mathbf{0}, \omega ; m), \tilde{B}(\mathbf{0}, \omega ; \sqrt{\alpha} m)$, and $\tilde{B}^{0}(\mathbf{0}, \omega ; \sqrt{\alpha} m)$, which can be confirmed by retaining the next-to-leading term in Eqs. (C7) and (C15). The contribution from the real parts are much smaller than these terms.
} 


$$
\begin{aligned}
\operatorname{Im} \Sigma_{+}(\mathbf{0}, \omega)= & \frac{g^{2}}{64 \pi m^{2}}\left[\omega^{3}\left(\tanh \frac{\omega^{2}}{4 T \omega}+\operatorname{coth} \frac{\omega^{2}}{4 T \omega}\right)\right. \\
& -\frac{\left(\omega^{2}+2 m^{2}\right)\left(\omega^{2}-m^{2}\right)^{2}}{\omega^{3}} \\
& \left.\times\left(\operatorname{coth} \frac{\omega^{2}+m^{2}}{4 T \omega}+\tanh \frac{\omega^{2}-m^{2}}{4 T \omega}\right)\right] \\
\simeq & \frac{1}{16 \pi} g^{2} T,
\end{aligned}
$$

where the first line is obtained by substituting $\mathbf{p}=0$ and $\alpha=0$ in Eq. (B2). In the second line the inequality $T \gg$ $\omega \gg m$ was used. Although this is positive and apparently breaks the analyticity, this order of the coupling should not be determined in the one-loop order, because the two-loop diagrams contain contributions of order $g^{2} T$. In fact, the analyticity problem can be cured by taking into account the two-loop diagrams.

\section{B. $1 / g^{2} \gg \alpha \lambda \gg 1$ (case 2$)$

$$
\text { 1. } \alpha \ll 1 /(g \lambda) \text { (case 2-a) }
$$

Due to the order estimate for $C_{2}(\omega), C_{4}(\omega)$, and $C_{6}(\omega)$ given in Eq. (4.19), we obtain

$$
C_{2}(\omega) \sim g\left(\frac{1}{\lambda}\right), \quad C_{4}(\omega) \sim g \alpha, \quad C_{6}(\omega) \sim\left(\frac{1}{\lambda}\right)
$$

which is the same as Eq. (4.22). The leading term of $C_{6}(\omega)$ cancels out with that of $C_{7}(\omega)$, and the remaining terms are of the order of $g \alpha,{ }^{7}$ while $C_{2}(\omega)$ and $C_{3}(\omega)$ do not. In the present case, however, $C_{4}(\omega)$ is larger than $C_{2}(\omega)$ and $C_{3}(\omega)$, and hence $C_{4}(\omega)$ dominates the gauge-dependent terms, which should be made small. This smallness is guaranteed when $\alpha \ll 1 / g$, which defines the adequate gauge-parameter region; this coincides with that in case 1. Notice that the inequality $g<\lambda$ must be assumed in this case, otherwise the inequality $\alpha<1 / g$ cannot be satisfied.

\section{2. $\alpha \gg 1 /(g \lambda)$ (case 2-b)}

Again, due to the order estimate in Eq. (4.19), $C_{2}(\omega)$, $C_{4}(\omega)$, and $C_{6}(\omega)$ are estimated to be

$C_{2}(\omega) \sim \frac{1}{\alpha}\left(\frac{1}{\lambda}\right)^{2}, \quad C_{4}(\omega) \sim\left(\frac{1}{\lambda}\right), \quad C_{6}(\omega) \sim \frac{1}{\alpha^{2} g^{2} \lambda^{3}}$.

Though $C_{4}(\omega)$ and $C_{7}(\omega)$ have the same order of magnitude, they do not cancel out, which can be confirmed from Eqs. (C20) and (C26). Therefore the largest contribution is $C_{4}(\omega) \sim 1 / \lambda \gg 1$; the present region for the gauge parameter $(\alpha \gg 1 /(g \lambda))$ is not an adequate gauge-parameter region.

\footnotetext{
${ }^{7}$ This term comes from $\operatorname{Im} \tilde{B}^{0}(\mathbf{0}, \omega ; \sqrt{\alpha} m)$, which can be confirmed from Eq. (C30). The real parts are negligible compared with this term.
}

\section{C. $\alpha \lambda \gg 1 / g^{2}$ (case 3)}

Here we treat the case where the gauge parameter is far larger than $1 / \lambda g^{2}$; this case includes the unitary gauge $(\alpha \rightarrow \infty)$. Due to the order estimate in Eq. (4.20) for this case, $C_{2}(\omega), C_{4}(\omega)$, and $C_{6}(\omega)$ are estimated to be

$$
C_{2}(\omega) \sim \frac{1}{\alpha}\left(\frac{1}{\lambda}\right)^{2}, \quad C_{4}(\omega) \sim\left(\frac{1}{\lambda}\right), \quad C_{6}(\omega) \sim \frac{1}{g^{2} \alpha^{2} \lambda^{3}} .
$$

Again, although $C_{4}(\omega)$ and $C_{7}(\omega)$ have the same order of magnitude, they do not actually cancel out due to the difference in the coefficients, which can be seen from Eqs. (C20) and (C42). In this case, the largest contribution is $C_{4}(\omega) \sim 1 / \lambda \gg 1$; therefore, this region is not an adequate gauge-parameter region. This also suggests that the order of the pole in the unitary gauge is not $g T$.

So let us now discuss the pole in the unitary gauge. First we assume the pole is of order $m$ instead of $g T$. Using Eqs. (4.20), (C50), and (C54), we have the following order estimates:

$$
\begin{aligned}
& C_{1}(\omega) \sim \frac{1}{\lambda}, \quad C_{2}(\omega) \sim-\frac{1}{\alpha \lambda}, \quad C_{3}(\omega) \sim \frac{g}{\sqrt{\lambda}}, \\
& C_{4}(\omega) \sim \frac{1}{\lambda}, \quad C_{5}(\omega) \sim \frac{g}{\sqrt{\lambda}}, \quad C_{6}(\omega) \sim\left(\frac{1}{\alpha g \lambda}\right)^{2}, \\
& C_{7}(\omega) \sim-\frac{1}{\lambda}
\end{aligned}
$$

For $\alpha \rightarrow \infty, C_{2}(\omega)$ and $C_{6}(\omega)$ vanish. Furthermore, $C_{3}(\omega)$ and $C_{5}(\omega)$ can be also neglected, because $g \sqrt{\lambda}=$ $m / T \ll 1$. The remaining parts $C_{1}(\omega), C_{4}(\omega)$ and $C_{7}(\omega)$ are estimated more precisely with the use of Eqs. (C20) and (C42), as follows:

$$
\begin{aligned}
& C_{1}(\omega) \simeq \frac{1}{8 \lambda} \frac{m^{2}}{\omega^{2}}, \quad C_{4}(\omega) \simeq \frac{1}{24 \lambda}, \\
& C_{7}(\omega) \simeq-\frac{1}{16 \lambda} .
\end{aligned}
$$

Collecting these terms, we reach at

$$
C(\omega) \simeq \frac{1}{48 \lambda}\left(\frac{6 m^{2}}{\omega^{2}}-1\right)
$$

This is precisely the same as the result obtained in the Proca formalism in the high temperature limit [8]; the thermal mass in this case reads

$$
\omega=\frac{\sqrt{6} m}{\sqrt{1+48 \lambda}} \stackrel{\lambda \rightarrow 0}{\rightarrow} \sqrt{6} m
$$

which can be seen from Fig. 10 also. We have confirmed numerically that the spectral function also approaches that in the Proca formalism as $\alpha \rightarrow \infty$. 


\section{Brief summary}

Let us summarize and discuss the results obtained so far in the preceding subsections for the gauge-parameter dependence of the fermion propagator at high temperature. The results are summarized in Fig. 11. We have found an adequate gauge-parameter region as $\alpha \ll 1 / g$ in which possible gauge dependence is of higher order of the couplings and hence can be neglected. We remark that this parameter restriction should also apply to QED.

In fact, the electron self-energy in the HTL approximation in QED at the next-to-leading level in the one-loop order reads $[22,23]$

$$
\begin{aligned}
\Sigma^{\mathrm{R}}(\mathbf{p}=\mathbf{0}, \omega) \simeq & \frac{e^{2} T^{2}}{8 \omega} \gamma^{0}+\frac{e^{2}}{8 \pi^{2}} \gamma^{0}\left(\omega \ln \frac{T}{\omega}-i \pi T\right) \\
& +(1-\alpha) \frac{e^{2}}{8 \pi^{2}} \gamma^{0}\left(-\omega \ln \frac{T}{\omega}+\frac{3 \pi i T}{2}\right) .
\end{aligned}
$$

If $\omega \sim e T$ and $\alpha \sim 1 / e$, the third term would be the same order as the leading term, which implies that the gauge independence is badly broken. Conversely, if $\alpha$ is much smaller than $1 / e$, then the gauge-dependent part becomes of order $e^{2}$ and can be neglected; this gives an adequate gauge-parameter region for QED.

There is a difference between QED and the massive vector theory in the Stueckelberg formalism: The limit $\alpha \rightarrow \infty$ cannot be taken in the former case because the third term in Eq. (4.31) diverges, while it can in the latter case. In this limit, the pole of the fermion propagator in the latter case becomes of order $m \ll g T$.

On the other hand, small $\alpha$ also yields the problem; the imaginary part of the fermion self-energy becomes positive for $\alpha \ll 1$, which breaks the analyticity of the self-energy.

The $\alpha$ dependence of the self-energy can be understood intuitively as follows: In the Stueckelberg formalism, there are two masses: the physical mass $m$ and the unphysical mass $\sqrt{\alpha} m$. For $g T \gg m, \sqrt{\alpha} m$, the self-energy naturally approaches that of QED because both masses can be neglected. By contrast, if the unphysical mass $\sqrt{\alpha} m$ is not smaller than $g T, \sqrt{\alpha} m$ cannot be neglected, although the temperature is high enough compared with the physical mass $m$. In this case, the self-energy at the one-loop level does not approach that of QED.

We also note here that in the $g \ll \lambda$ case, the boson mass $m$ cannot be taken to zero from the outset, but the selfenergy is approximately equal to that in the HTL approximation in QED as long as $\alpha \ll 1 / g$. This implies that the value, $T g^{3 / 2}$, is the upper limit of the boson mass that we can neglect.

Summarizing the situation, we see that there are three regions of the gauge parameter: In the first region, the theory approaches QED, and is in an adequate gaugeparameter region. In the second region, the theory approaches QED, but is out of an adequate gauge-parameter region. In the third region, the theory does not approach QED, and is out of an adequate gauge-parameter region. We conclude that the gauge parameter should be chosen to be $1 \lesssim \alpha \ll 1 / g$ in numerical calculations in this formalism.

\section{SUMMARY AND CONCLUDING REMARKS}

We have investigated the spectral properties of a fermion coupled with a massive vector boson in the whole temperature $(T)$ region at one-loop order. The vector boson with a mass $(m)$ is introduced as a $U(1)$ gauge boson in the Stueckelberg formalism so that the high $T$ limit, or equivalently the massless limit in the sense that $m / T \rightarrow 0$, can be taken ${ }^{8}$ : We have successfully analyzed and clarified the characteristics of the spectral properties of the fermion in the distinct three regions of $T$, i.e., (I) $T \ll m$, (II) $T \sim m$, and (III) $T \gg m$ regions, in a unified way. We have also carefully examined the possible gauge dependence of the spectral properties of the fermion in the respective three $T$ regions, separately.

In region (I), the fermion spectral properties hardly change from those in the vacuum, which are gauge independent. In region (II), the fermion spectral function gets to have a three-peak structure in the small momentum region with supports in the positive, zero, and negative energy regions; the three-peak structure becomes prominent when $T \simeq 2 m$ for $g=0.5$. We have confirmed numerically that the fermion poles and hence the fermion spectral function shows virtually no dependence on the gauge parameter $(\alpha)$ for $T \sim m$. It is thus natural that the similar three-peak structure of the fermion spectral function was obtained in the Proca formalism for the massive vector field for $T \sim m$ [8], since the Proca formalism exactly corresponds to the unitary gauge $\alpha \rightarrow \infty$. Conversely, the three-peak structure found in [8] is not an artifact by a special choice of the gauge and is physical.

It is interesting that the spectral function of a fermion coupled with a scalar massive boson also shows a similar three-peak structure for $T \sim m$ at the one-loop level $[8,9]$. The present analysis has established that a fermion coupled with a massive boson with a mass $m$ has a three-peak structure for small momenta with supports in the positive, vanishing, and negative energy regions at temperatures comparable with the boson mass, irrespective of the type of the boson, at the one-loop order.

For $T \gg m$ [region (III)], the fermion spectral function tends to have distinct two peaks precisely corresponding to those seen in QED in the HTL approximation [1-4]. It means that our formalism nicely describes the spectral function of the fermion coupled with a massive vector boson even in the high $T$ region. There is, however, a tricky point related to a possible gauge dependence. We have

\footnotetext{
${ }^{8}$ As is mentioned in Introduction, the Proca formalism does not yield sensible results for $T \gg m[8,18,19]$.
} 
found that there exists an adequate region of the gauge parameter $\alpha$ in the high- $T$ region for the perturbation theory at finite $T$ : If $\alpha$ is of the order 1 , the analysis at the one-loop order does not create a problem and is reliable, keeping the positivity of the spectral function and so on; otherwise, however, these fundamental properties may be lost. This is because two mass parameters exist, i.e., the vector-boson mass $m$ and the ghost mass $\sqrt{\alpha} m$ which is inherently in the Stueckelberg formalism. Thus, the precise high- $T$ region should be defined by the two conditions, $T \gg m$ and $T \gg \sqrt{\alpha} m$. Our extensive analytic study has proven this observation and shown that when $\alpha \ll 1 / g$ ( $g$ is the coupling constant), the one-loop analysis is reliable even in the region (III). Accordingly, if the unitary gauge $(\alpha \rightarrow \infty)$ is adopted for the massive vector boson, the one-loop analysis cannot be valid for $T \gg m$, as is shown in a different context $[18,19]$.

Our numerical calculation has shown that there still remains a peak at the origin in the $\omega-|\mathbf{p}|$ plane, though with a faint strength even in region (III); this is in contrast to QED in the HTL approximation where such a peak is absent. Although it is an interesting possibility that the three-peak structure persists at the high- $T$ region and even in QED, a sensible analysis of the spectral properties around such a low-energy region requires a resummed perturbation theory to deal with possible pinch singularities [20]. Thus, we leave an analysis of the spectral properties in the very low-energy region to a future work and hope to report it elsewhere [21].

As was mentioned in the Introduction, we can think of some physical situations where the present analysis can be relevant, since massive vector bosons appear at finite $T$ in various physical systems. In $\mathrm{QCD}$, vector bosons or vectorbosonic modes may decrease their masses in association with the restoration of chiral symmetry at finite $T$ [13]. It would be not surprising if vector-bosonic modes exist even in the deconfined and chiral symmetric phase in the vicinity of the critical temperature $T_{c}$, since the existence of other hadronic modes $[7,24,25]$ and bound states [26] are suggested in that temperature region. A vector-type glue ball may also exist in such a system. Then the present analysis would suggest that the quark spectra can be largely affected in such a system where the boson mass is comparable to $T$ in the order of magnitude. In the electroweak theory, the dispersion relation of neutrinos at high $T$ may possibly be affected by the weak bosons the masses of which change with $T$ [14]. One of the findings of the present analysis tells us that the one-loop analysis in the unitary gauge cannot be applicable when $m / T \ll 1$, which includes the vicinity of the critical point.

\section{ACKNOWLEDGMENTS}

We thank M. Kitazawa and M. Harada for helpful comments and discussions. This work was supported by the Grant-in-Aid for the Global COE Program "The Next
Generation of Physics, Spun from Universality and Emergence" from the Ministry of Education, Culture, Sports, Science and Technology (MEXT) of Japan and by a Grant-in-Aid for Scientific Research by the Ministry of Education, Culture, Sports, Science and Technology (MEXT) of Japan under Contract Nos. 20540265 and 19.07797 .

\section{APPENDIX A: THE CORRESPONDENCE BETWEEN THE STUECKELBERG FORMALISM AND THE ABELIAN-HIGGS MODEL}

In this Appendix, we briefly show that the AbelianHiggs model is reduced to the $U(1)$ gauge theory with a massive gauge boson in the Stueckelberg formalism [11,17].

The Lagrangian of the Abelian-Higgs model reads

$$
\mathcal{L}_{\text {Higgs }}=-\frac{1}{4} F^{\mu \nu} F_{\mu \nu}+\left|\left(\partial_{\mu}-i e A_{\mu}\right) \Phi\right|^{2},
$$

with $F_{\mu \nu}=\partial_{\mu} A_{\nu}-\partial_{\nu} A_{\mu}$. Here $A_{\mu}$ and $\Phi$ denote the vector and the Higgs field, respectively.

We fix the absolute value of the Higgs field $\Phi$ and use the following polar representation:

$$
\Phi=\frac{m}{e \sqrt{2}} \exp \left(\frac{i e B(x)}{m}\right),
$$

with $|\Phi|=m /(e \sqrt{2})$. We remark that the scalar field $B$, which will turn to be identified with the Stueckelberg field, is introduced as the phase of $\Phi$. Then, the Lagrangian becomes

$$
\mathcal{L}_{\text {Higgs }}=-\frac{1}{4} F^{\mu \nu} F_{\mu \nu}+\frac{m^{2}}{2}\left(A^{\mu}-\frac{1}{m} \partial^{\mu} B\right)^{2},
$$

which exactly gives the free Lagrangian of the massive vector field in the Stueckelberg formalism and its interaction term with the Stueckelberg field $B$ given in Eq. (2.1) in the text. This is what we wanted to show.

\section{APPENDIX B: CALCULATION OF THE FERMION SELF-ENERGY}

Here we derive Eq. (2.21) in the text. We first recall that the retarded self-energy $\Sigma^{\mathrm{R}}(\mathbf{p}, \omega)$ in the one-loop approximation is given from Eq. (2.18) by the analytic continuation, $i \omega_{m} \rightarrow \omega+i \epsilon \equiv p^{0}$; see Eq. (2.10). Then its imaginary part reads

$$
\begin{aligned}
\operatorname{Im} & \Sigma^{\mathrm{R}}(\mathbf{p}, \omega)=-2 g^{2} \gamma^{\mu} \operatorname{Im} \tilde{B} \tilde{\mu}_{\mu}(\mathbf{p}, \omega ; m) \\
& +\frac{g^{2}}{m^{2}}\left[p \left(p^{2}(\operatorname{Im} \tilde{B}(\mathbf{p}, \omega ; \sqrt{\alpha} m)-\operatorname{Im} \tilde{B}(\mathbf{p}, \omega ; m))\right.\right. \\
& \left.-m^{2}(\alpha \operatorname{Im} \tilde{B}(\mathbf{p}, \omega ; \sqrt{\alpha} m)-\operatorname{Im} \tilde{B}(\mathbf{p}, \omega ; m))\right) \\
& \left.-p^{2} \gamma^{\mu}\left(\operatorname{Im} \tilde{B}_{\mu}(\mathbf{p}, \omega ; \sqrt{\alpha} m)-\operatorname{Im} \tilde{B}_{\mu}(\mathbf{p}, \omega ; m)\right)\right],
\end{aligned}
$$

where $p^{2}=\omega^{2}-|\mathbf{p}|^{2}$. By applying the projection operator for the particle sector, we have 


$$
\begin{aligned}
\operatorname{Im} \Sigma_{+}(\mathbf{p}, \omega)= & \frac{1}{2} \operatorname{Tr}\left[\operatorname{Im} \Sigma^{\mathrm{R}}(\mathbf{p}, \omega) \Lambda_{+}(\mathbf{p}) \gamma^{0}\right] \\
= & -2 g^{2}\left(\operatorname{Im} \tilde{B}^{0}(\mathbf{p}, \omega ; m)-\hat{\mathbf{p}}_{i} \operatorname{Im} \tilde{B}_{i}(\mathbf{p}, \omega ; m)\right)+\frac{g^{2}}{m^{2}}\left[( \omega - | \mathbf { p } | ) \left(p^{2}(\operatorname{Im} \tilde{B}(\mathbf{p}, \omega ; \sqrt{\alpha} m)-\operatorname{Im} \tilde{B}(\mathbf{p}, \omega ; m))\right.\right. \\
& \left.-m^{2}(\alpha \operatorname{Im} \tilde{B}(\mathbf{p}, \omega ; \sqrt{\alpha} m)-\operatorname{Im} \tilde{B}(\mathbf{p}, \omega ; m))\right)-p^{2}\left(\operatorname{Im} \tilde{B}^{0}(\mathbf{p}, \omega ; \sqrt{\alpha} m)-\operatorname{Im} \tilde{B}^{0}(\mathbf{p}, \omega ; m)\right. \\
& \left.\left.-\hat{\mathbf{p}}_{i} \operatorname{Im} \tilde{B}_{i}(\mathbf{p}, \omega ; \sqrt{\alpha} m)+\hat{\mathbf{p}}_{i} \operatorname{Im} \tilde{B}_{i}(\mathbf{p}, \omega ; m)\right)\right] .
\end{aligned}
$$

Here we have used the relation $\operatorname{Tr}\left[p \Lambda_{+}(\mathbf{p}) \gamma^{0}\right] / 2=\omega-$ $|\mathbf{p}| \quad$ and $\operatorname{Tr}\left[\tilde{B}^{\mu}(\mathbf{p}, \omega ; m) \gamma_{\mu} \Lambda_{+}(\mathbf{p}) \gamma^{0}\right]=\tilde{B}^{0}(\mathbf{p}, \omega ; m)-$ $\hat{\mathbf{p}}_{i} \tilde{B}_{i}(\mathbf{p}, \omega ; m)$. Notice that $\operatorname{Im} \tilde{B}(\mathbf{p}, \omega ; m)$ and $\operatorname{Im} \tilde{B}^{\mu}(\mathbf{p}, \omega ; m)$ contain $\delta\left(s E_{f}-t E_{b}-\omega\right)$ with $s, t=$ $\pm 1, \quad E_{f}=|\mathbf{k}|, \quad$ and $\quad E_{b}=\sqrt{m^{2}+(\mathbf{p}-\mathbf{k})^{2}}, \quad$ while $\operatorname{Im} \tilde{B}(\mathbf{p}, \omega ; \sqrt{\alpha} m)$ and $\operatorname{Im} \tilde{B}^{\mu}(\mathbf{p}, \omega ; \sqrt{\alpha} m)$ contain $\delta\left(s E_{f}-\right.$ $\left.t E_{b}^{\prime}-\omega\right)$ with $E_{b}^{\prime}=\sqrt{\alpha m^{2}+(\mathbf{p}-\mathbf{k})^{2}}$. Collecting the terms which contain $\delta\left(s E_{f}-t E_{b}-\omega\right)$, we have

$$
\begin{aligned}
& \left(\delta\left(s E_{f}-t E_{b}-\omega\right) \text { term }\right) \\
& =g^{2} \int \frac{d^{3} \mathbf{k}}{(2 \pi)^{3}} \sum_{s, t= \pm 1} \frac{s t \pi}{4 E_{f} E_{b}} \delta\left(s E_{f}-t E_{b}-\omega\right) \\
& \quad \times\left(f\left(s E_{f}\right)+n\left(s E_{f}-\omega\right)\right)\left(-2\left(s E_{f}-\hat{\mathbf{p}} \cdot \mathbf{k}\right)\right. \\
& \left.\quad-\frac{1}{m^{2}}(\omega-|\mathbf{p}|)\left(p^{2}-m^{2}\right)+\frac{1}{m^{2}} p^{2}\left(s E_{f}-\hat{\mathbf{p}} \cdot \mathbf{k}\right)\right) .
\end{aligned}
$$

Here we have used the following formulae;

$$
\begin{aligned}
\operatorname{Im} \tilde{B}(\mathbf{p}, \omega ; m)= & \int \frac{d^{3} \mathbf{k}}{(2 \pi)^{3}} \sum_{s, t= \pm 1} \frac{s t \pi}{4 E_{f} E_{b}} \delta\left(s E_{f}-t E_{b}-\omega\right) \\
& \times\left(f\left(s E_{f}\right)+n\left(s E_{f}-\omega\right)\right), \\
\operatorname{Im} \tilde{B}^{0}(\mathbf{p}, \omega ; m)= & \int \frac{d^{3} \mathbf{k}}{(2 \pi)^{3}} \sum_{s, t= \pm 1} \frac{s t \pi}{4 E_{f} E_{b}} \delta\left(s E_{f}-t E_{b}-\omega\right) \\
& \times\left(f\left(s E_{f}\right)+n\left(s E_{f}-\omega\right)\right) s E_{f}, \\
\operatorname{Im} \tilde{B}^{i}(\mathbf{p}, \omega ; m)= & \int \frac{d^{3} \mathbf{k}}{(2 \pi)^{3}} \sum_{s, t= \pm 1} \frac{s t \pi}{4 E_{f} E_{b}} k^{i} \delta\left(s E_{f}-t E_{b}-\omega\right) \\
& \times\left(f\left(s E_{f}\right)+n\left(s E_{f}-\omega\right)\right) .
\end{aligned}
$$

Using the constraint $\hat{\mathbf{p}} \cdot \mathbf{k}=E_{f} \cos \theta=\left(m^{2}-p^{2}+\right.$ $\left.2 \omega s E_{f}\right) / 2|\mathbf{p}|$ posed by the delta function, we have

$$
\begin{aligned}
\left(\delta\left(s E_{f}-t E_{b}-\omega\right) \text { term }\right)= & g^{2} \int \frac{d^{3} \mathbf{k}}{(2 \pi)^{3}} \sum_{s, t= \pm 1} \frac{s t \pi}{4 E_{f} E_{b}} \delta\left(s E_{f}-t E_{b}-\omega\right)\left(f\left(s E_{f}\right)+n\left(s E_{f}-\omega\right)\right) \\
& \times\left(\frac{p^{2}-m^{2}}{2|\mathbf{p}| m^{2}}\left((\omega-|\mathbf{p}|)^{2}-2 m^{2}\right)+s E_{f} \frac{2 m^{2}-p^{2}}{m^{2}|\mathbf{p}|}(\omega-|\mathbf{p}|)\right) \\
= & \frac{g^{2}}{32 \pi m^{2}|\mathbf{p}|^{2}} \int_{0}^{\infty} d E_{f} \sum_{s, t= \pm 1} s t\left[\left(p^{2}-m^{2}\right)\left((\omega-|\mathbf{p}|)^{2}-2 m^{2}\right)+2 s E_{f}(\omega-|\mathbf{p}|)\left(2 m^{2}-p^{2}\right)\right] \\
& \times\left(f\left(s E_{f}\right)+n\left(s E_{f}-\omega\right)\right) \int_{e_{-}}^{e_{+}} d E_{b} \delta\left(s E_{f}-t E_{b}-\omega\right),
\end{aligned}
$$

with $e_{ \pm} \equiv \sqrt{\left(|\mathbf{p}| \pm E_{f}\right)^{2}+m^{2}}$. Here the momentum integral is converted to that for the particle energies through the formula

$$
\int d^{3} \mathbf{k}=\frac{2 \pi}{|\mathbf{p}|} \int_{0}^{\infty} d E_{f} E_{f} \int_{e_{-}}^{e_{+}} d E_{b} E_{b} .
$$

We now evaluate the integral separately for the timelike and the spacelike region. For the timelike region $(|\omega|>$ $|\mathbf{p}|)$, we have

$$
\begin{aligned}
& \left(\delta\left(s E_{f}-t E_{b}-\omega\right) \text { term }\right)=\frac{g^{2}}{32 \pi|\mathbf{p}|^{2} m^{2}} \int_{E_{f}^{+}}^{E_{f}^{-}} d E_{f}\left(f\left(E_{f}\right)\right. \\
& \left.\quad+n\left(E_{f}-\omega\right)\right)\left[\left(-p^{2}+m^{2}\right)\left((|\mathbf{p}|-\omega)^{2}-2 m^{2}\right)\right. \\
& \left.\quad+2\left(p^{2}-2 m^{2}\right) E_{f}(\omega-|\mathbf{p}|)\right]
\end{aligned}
$$

with $E_{f}^{ \pm} \equiv\left(p^{2}-m^{2}\right) /(2(\omega \pm|\mathbf{p}|))$. For the spacelike region $(|\omega|<|\mathbf{p}|)$, we have 


$$
\begin{aligned}
& \left(\delta\left(s E_{f}-t E_{b}-\omega\right) \operatorname{term}\right)=\frac{g^{2}}{32 \pi|\mathbf{p}|^{2} m^{2}} \int_{E_{f}^{+}}^{E_{f}^{-}} d E_{f}\left(f\left(E_{f}\right)\right. \\
& \left.\quad+n\left(E_{f}-\omega\right)\right)\left[\left(-p^{2}+m^{2}\right)\left((|\mathbf{p}|-\omega)^{2}-2 m^{2}\right)\right. \\
& \left.\quad+2\left(p^{2}-2 m^{2}\right) E_{f}(\omega-|\mathbf{p}|)\right]-\frac{g^{2}}{32 \pi|\mathbf{p}|^{2} m^{2}} \\
& \quad \times\left[\left(p^{2}-2 m^{2}\right)(\omega-|\mathbf{p}|) \pi^{2} T^{2}\right. \\
& \left.\quad+\omega\left[2 m^{4}-p^{2}\left(|\mathbf{p}|(\omega-|\mathbf{p}|)+m^{2}\right)\right]\right] .
\end{aligned}
$$

Here we have used the integral formulae

$$
\int_{-\infty}^{\infty} d E_{f}\left(f\left(E_{f}\right)+n\left(E_{f}-\omega\right)\right)=-\omega
$$

$$
\int_{-\infty}^{\infty} d E_{f}\left(f\left(E_{f}\right)+n\left(E_{f}-\omega\right)\right) E_{f}=\frac{\pi^{2} T^{2}}{2}-\frac{\omega^{2}}{2} .
$$

Combining Eqs. (B9) and (B10), we have

$$
\begin{aligned}
& \left(\delta\left(s E_{f}-t E_{b}-\omega\right) \text { term }\right)=\frac{g^{2}}{32 \pi|\mathbf{p}|^{2} m^{2}} \int_{E_{f}^{+}}^{E_{f}^{-}} d E_{f}\left(f\left(E_{f}\right)\right. \\
& \left.\quad+n\left(E_{f}-\omega\right)\right)\left[\left(-p^{2}+m^{2}\right)\left((|\mathbf{p}|-\omega)^{2}-2 m^{2}\right)\right. \\
& \left.\quad+2\left(p^{2}-2 m^{2}\right) E_{f}(\omega-|\mathbf{p}|)\right]-\frac{g^{2}}{32 \pi|\mathbf{p}|^{2} m^{2}} \theta\left(-p^{2}\right) \\
& \quad \times\left[\left(p^{2}-2 m^{2}\right)(\omega-|\mathbf{p}|) \pi^{2} T^{2}\right. \\
& \left.\quad+\omega\left[2 m^{4}-p^{2}\left(|\mathbf{p}|(\omega-|\mathbf{p}|)+m^{2}\right)\right]\right] .
\end{aligned}
$$

Next, we collect the terms that contain $\delta\left(s E_{f}-\right.$ $\left.t E_{b}^{\prime}-\omega\right)$

$$
\begin{aligned}
\left(\delta\left(s E_{f}-t E_{b}^{\prime}-\omega\right) \text { term }\right)= & \left.\frac{g^{2}}{32 \pi m^{2}|\mathbf{p}|^{2}} \int_{0}^{\infty} d E_{f} \sum_{s, t= \pm 1} s t(\omega-|\mathbf{p}|)\left[-(\omega-|\mathbf{p}|)\left(p^{2}-\alpha m^{2}\right)+2 p^{2} s E_{f}\right)\right] \\
& \times\left(f\left(s E_{f}\right)+n\left(s E_{f}-\omega\right)\right) \int_{e_{-}^{\prime}}^{e_{+}^{\prime}} d E_{b}^{\prime} \delta\left(s E_{f}-t E_{b}^{\prime}-\omega\right) \\
= & -\frac{g^{2}}{32 \pi|\mathbf{p}|^{2} m^{2}} \int_{E_{f}^{\prime+}}^{E_{f}^{\prime-}} d E_{f}\left(f\left(E_{f}\right)+n\left(E_{f}-\omega\right)\right)\left[\left(-p^{2}+m^{2} \alpha\right)(|\mathbf{p}|-\omega)^{2}+2 p^{2} E_{f}(\omega-|\mathbf{p}|)\right] \\
& +\frac{g^{2}}{32 \pi|\mathbf{p}|^{2} m^{2}} \theta\left(-p^{2}\right)\left[p^{2}(\omega-|\mathbf{p}|) \pi^{2} T^{2}+\omega(\omega-|\mathbf{p}|)\left(-\omega p^{2}-(\omega-|\mathbf{p}|)\left(-p^{2}+m^{2} \alpha\right)\right)\right]
\end{aligned}
$$

where $\quad e_{ \pm}^{\prime} \equiv \sqrt{\left(|\mathbf{p}| \pm E_{f}\right)^{2}+\alpha m^{2}} \quad$ and $\quad E_{f}^{\prime \pm} \equiv\left(p^{2}-\right.$ $\left.\alpha m^{2}\right) /(2(\bar{\omega} \pm|\mathbf{p}|))$. Combining Eqs. (B13) and (B14), we arrive at Eq. (2.21).

\section{APPENDIX C: POWER COUNTING OF THE FERMION SELF-ENERGY}

In this Appendix, we make an order estimate of $\tilde{B}(\mathbf{p}, \omega ; m)$ and $\tilde{B}^{0}(\mathbf{p}, \omega ; m)$ used in the analysis done in Sec. IV. The estimate will be made separately for the following four limiting cases: $T \gg \omega \gg m, T \gg m \gg$ $\omega, m \gg T \gg \omega$, and $T \gg \omega \sim m$. For simplicity, we set $|\mathbf{p}|=0$.

We start with $\tilde{B}\left(\mathbf{0}, i \omega_{m} ; m\right)$ and $\tilde{B}^{0}\left(\mathbf{0}, i \omega_{m} ; m\right)$ in the imaginary time formalism:

$$
\begin{aligned}
\tilde{B}\left(\mathbf{0}, i \omega_{m} ; m\right)= & \int \frac{d^{3} \mathbf{k}}{(2 \pi)^{3}} \sum_{s, t= \pm 1} \frac{s t}{4 E_{b} E_{f}} \frac{1}{s E_{f}-t E_{b}-i \omega_{m}} \\
& \times\left(f\left(s E_{f}\right)+n\left(t E_{b}\right)\right),
\end{aligned}
$$

$$
\begin{array}{r}
\tilde{B}^{0}\left(\mathbf{0}, i \omega_{m} ; m\right)=\int \frac{d^{3} \mathbf{k}}{(2 \pi)^{3}} \sum_{s, t= \pm 1} \frac{s t}{4 E_{b} E_{f}} \\
\times \frac{s E_{f} f\left(s E_{f}\right)+\left(t E_{b}+i \omega_{m}\right) n\left(t E_{b}\right)}{s E_{f}-t E_{b}-i \omega_{m}} .
\end{array}
$$

Performing the analytic continuation and dropping $T=0$ part, we get the corresponding retarded functions, the real parts of which read

$$
\begin{aligned}
& \operatorname{Re} \tilde{B}(\mathbf{0}, \omega ; m)_{T \neq 0} \\
& =\mathrm{P} \int \frac{d^{3} \mathbf{k}}{(2 \pi)^{3}} \sum_{s, t= \pm 1} \frac{s t}{4 E_{b} E_{f}} \frac{1}{s E_{f}-t E_{b}-\omega}\left(f\left(s E_{f}\right)+n\left(t E_{b}\right)\right) \\
& \quad-\operatorname{Re} \tilde{B}(\mathbf{0}, \omega ; m)_{T=0} \\
& =\frac{1}{2 \pi^{2}} \mathrm{P} \int_{0}^{\infty} d E_{f}\left(E_{f} f\left(E_{f}\right) \frac{\omega^{2}-m^{2}}{\left(\omega^{2}-m^{2}\right)^{2}-4 E_{f}^{2} \omega^{2}}\right. \\
& \left.\quad-n\left(E_{b}\right) \frac{E_{f}^{2}}{E_{b}} \frac{m^{2}+\omega^{2}}{\left(\omega^{2}-m^{2}\right)^{2}-4 E_{f}^{2} \omega^{2}}\right),
\end{aligned}
$$




$$
\begin{aligned}
& \operatorname{Re} \tilde{B}^{0}(\mathbf{0}, \omega ; m)_{T \neq 0} \\
& =\mathrm{P} \int \frac{d^{3} \mathbf{k}}{(2 \pi)^{3}} \sum_{s, t= \pm 1} \frac{s t}{4 E_{b} E_{f}} \frac{s E_{f} f\left(s E_{f}\right)+\left(t E_{b}+\omega\right) n\left(t E_{b}\right)}{s E_{f}-t E_{b}-\omega} \\
& \quad-\operatorname{Re} \tilde{B}^{0}(\mathbf{0}, \omega ; m)_{T=0} \\
& =\frac{\omega}{2 \pi^{2}} \mathrm{P} \int_{0}^{\infty} d E_{f}\left(2 E_{f}^{3} f\left(E_{f}\right) \frac{1}{\left(\omega^{2}-m^{2}\right)^{2}-4 E_{f}^{2} \omega^{2}}\right. \\
& \left.\quad+n\left(E_{b}\right) \frac{E_{f}^{2}}{E_{b}} \frac{2 E_{f}^{2}+m^{2}-\omega^{2}}{\left(\omega^{2}-m^{2}\right)^{2}-4 E_{f}^{2} \omega^{2}}\right) .
\end{aligned}
$$

Here we have defined $T \neq 0$ parts as $\tilde{B}(\mathbf{0}, \omega ; m)_{T \neq 0} \equiv$ $\tilde{B}(\mathbf{0}, \omega ; m)-\tilde{B}(\mathbf{0}, \omega ; m)_{T=0} \quad$ and $\quad \tilde{B}^{0}(\mathbf{0}, \omega ; m)_{T \neq 0} \equiv$ $\tilde{B}^{0}(\mathbf{0}, \omega ; m)-\tilde{B}^{0}(\mathbf{0}, \omega ; m)_{T=0}$. In the evaluation of these integrals, we have to carefully separate and deal with the contributions from the soft region $\left(E_{f} \ll T\right)$ and the hard region $\left(E_{f} \sim T\right)$ unlike the calculation of the leading order of the HTL approximation, because the contribution from the soft region can be much larger than that from the hard region in some limits.

From Eqs. (B4) and (B5), the finite temperature parts of $\operatorname{Im} \tilde{B}(\mathbf{0}, \omega ; m)$ and $\operatorname{Im} \tilde{B}^{0}(\mathbf{0}, \omega ; m)$ are calculated to be

$$
\begin{aligned}
& \operatorname{Im} \tilde{B}(\mathbf{0}, \omega ; m)_{T \neq 0} \\
& =\frac{1}{8 \pi} \int_{0}^{\infty} d E_{f} \frac{E_{f}}{E_{b}}\left[-\left(f\left(E_{f}\right)+n\left(E_{b}\right)\right) \delta\left(E_{f}-E_{b}+\omega\right)\right. \\
& \left.\quad-\left(f\left(E_{f}\right)-n\left(E_{b}\right)\right) \delta\left(E_{f}+E_{b}-\omega\right)\right] \\
& =\frac{\omega^{2}-m^{2}}{16 \pi \omega^{2}}\left(\operatorname{sgn}\left(m^{2}-\omega^{2}\right) f\left(\left|\frac{m^{2}-\omega^{2}}{2 \omega}\right|\right)\right. \\
& \left.\quad+n\left(\frac{m^{2}+\omega^{2}}{2 \omega}\right)\right)
\end{aligned}
$$

and

$$
\begin{aligned}
\operatorname{Im} & \tilde{B}^{0}(\mathbf{0}, \omega ; m)_{T \neq 0} \\
= & -\frac{1}{8 \pi} \int_{0}^{\infty} d E_{f} \frac{E_{f}^{2}}{E_{b}}\left[-\left(f\left(E_{f}\right)+n\left(E_{b}\right)\right) \delta\left(E_{f}-E_{b}+\omega\right)\right. \\
& \left.+\left(f\left(E_{f}\right)-n\left(E_{b}\right)\right) \delta\left(E_{f}+E_{b}-\omega\right)\right] \\
= & \frac{\left(\omega^{2}-m^{2}\right)^{2}}{32 \pi \omega^{3}}\left(\operatorname{sgn}\left(m^{2}-\omega^{2}\right) f\left(\left|\frac{m^{2}-\omega^{2}}{2 \omega}\right|\right)\right. \\
& \left.+n\left(\frac{m^{2}+\omega^{2}}{2 \omega}\right)\right),
\end{aligned}
$$

respectively. In the first lines, the positiveness of $\omega$ has been taken into account.

$$
\begin{gathered}
\text { 1. } \boldsymbol{T} \gg \omega \gg m \\
\text { a. } \tilde{B}(\mathbf{0}, \omega ; m)
\end{gathered}
$$
have

$$
\operatorname{Im} \tilde{B}(\mathbf{0}, \omega ; m)_{T \neq 0} \simeq \frac{1}{16 \pi}\left(-f\left(\frac{\omega}{2}\right)+n\left(\frac{\omega}{2}\right)\right)=\frac{1}{8 \pi} n(\omega) .
$$

Here we have utilized the inequality $\omega \gg m$. Using the approximate formula

$$
n(x) \simeq T / x \text { for } x \ll T,
$$

we get

$$
\operatorname{Im} \tilde{B}(\mathbf{0}, \omega ; m)_{T \neq 0} \simeq \frac{1}{8 \pi} \frac{T}{\omega} .
$$

The real part is given by Eq. (C3). To evaluate it, it is found convenient to introduce an intermediate scale $T^{*}$ which satisfies the relation $T \gg T^{*} \gg \omega$ so that $T^{*}$ separates the hard region from the soft region. The contribution from $E_{f}>T^{*}$, which is denoted by $\operatorname{Re} \tilde{B}(\mathbf{0}, \omega ; m)_{T \neq 0}^{E_{f}>T^{*}}$, is evaluated to be

$\operatorname{Re} \tilde{B}(\mathbf{0}, \omega ; m)_{T \neq 0}^{E_{f}>T^{*}} \simeq-\frac{1}{8 \pi^{2}} \mathrm{P} \int_{T^{*}}^{\infty} d E_{f} \frac{1}{E_{f}}\left(f\left(E_{f}\right)-n\left(E_{f}\right)\right)$.

Here we have used the inequalities $\omega \gg m$ and $E_{f}>$ $T^{*} \gg \omega$ in the hard region. We get the following expression by integrating by part:

$$
\begin{gathered}
\operatorname{Re} \tilde{B}(\mathbf{0}, \omega ; m)_{T \neq 0}^{E_{f}>T^{*}} \simeq-\frac{1}{8 \pi^{2}}\left(-\left[f\left(E_{f}\right)-n\left(E_{f}\right)\right]_{T^{*}}^{\infty}\right. \\
\left.\quad+\mathrm{P} \int_{T^{*}}^{\infty} d E_{f} \frac{1}{E_{f}} \frac{d}{d E_{f}} E_{f}\left(f\left(E_{f}\right)-n\left(E_{f}\right)\right)\right) .
\end{gathered}
$$

By inserting Eq. (C8) and $f(x) \simeq 1 / 2$ for $x \ll 1$, we see that the first term is of the order of $T / T^{*}$. It is easily confirmed that the second term is of the order of $\ln \left(T^{*} / T\right)$ by performing partial integration again, so the second term can be neglected;

$$
\operatorname{Re} \tilde{B}(\mathbf{0}, \omega ; m)_{T \neq 0}^{E_{f}>T^{*}} \simeq \frac{1}{8 \pi^{2}} \frac{T}{T^{*}} .
$$

On the other hand, we have for the contribution from $E_{f}<T^{*}$, which is denoted by $\operatorname{Re} \tilde{B}(\mathbf{0}, \omega ; m)_{T \neq 0}^{E_{f}<T^{*}}$ :

$$
\begin{aligned}
\operatorname{Re} \tilde{B}(\mathbf{0}, \omega ; m)_{T \neq 0}^{E_{f}<T^{*}} \simeq & -\frac{1}{2 \pi^{2}} \mathrm{P} \int_{0}^{T^{*}} d E_{f} \frac{T E_{f}^{2}}{E_{f}^{2}+m^{2}} \\
& \times \frac{\omega^{2}}{\omega^{4}-4 E_{f}^{2} \omega^{2}} .
\end{aligned}
$$

Here again, Eq. (C8) has been inserted and the inequality $\omega \gg m$ has been taken into account. The term which has $f\left(E_{f}\right)$ has been neglected since it is much smaller than $n\left(E_{b}\right)$. Performing partial fraction decomposition and utilizing the inequality $\omega \gg m$, we get

$$
\operatorname{Re} \tilde{B}(\mathbf{0}, \omega ; m)_{T \neq 0}^{E_{f}<T^{*}} \simeq-\frac{T}{8 \pi^{2} T^{*}}+\frac{T m}{4 \pi \omega^{2}} .
$$


We see that the imaginary part, Eq. (C9), is much larger than any term of the real part, Eqs. (C12) and (C14).

A remark is in order here: In the present analysis, we have considered only the leading term in the high temperature expansion as in [16,19], where the simple approximate formula $n(x) \simeq T / x$ is adopted. In fact, all the analyses given in this Appendix will be based on this formula. Of course, one could include the next-to-leading term by using the formula $n(x) \simeq T / x-1 / 2$, which would lead to the logarithmic terms derived in $[22,23]$. The inclusion of such terms is beyond the scope of this work.

$$
\text { b. } \tilde{B}^{0}(\mathbf{0}, \omega ; m)
$$

The imaginary part of $\tilde{B}^{0}(\mathbf{0}, \omega ; m)_{T \neq 0}$ is given by Eq. (C6), which is estimated to be

$$
\operatorname{Im} \tilde{B}^{0}(\mathbf{0}, \omega ; m)_{T \neq 0} \simeq \frac{T}{16 \pi} .
$$

The derivation of this expression is the same as that of Eq. (C9).

The real part is given by Eq. (C4). We introduce $T^{*}$ which satisfies $T \gg T^{*} \gg \omega$, as before. The contribution from the $E_{f}>T^{*}$ region, which is written as $\operatorname{Re} \tilde{B}^{0}(\mathbf{0}, \omega ; m)_{T \neq 0}^{E_{f}>T^{*}}$, is evaluated as follows by inserting $E_{b} \simeq E_{f}$ and $E_{f} \gg \omega \gg m$ again:

$$
\begin{aligned}
\operatorname{Re} \tilde{B}^{0}(\mathbf{0}, \omega ; m)_{T \neq 0}^{E_{f}>T^{*}} \simeq & \frac{\omega}{4 \pi^{2}} \int_{T^{*}}^{\infty} d E_{f} E_{f} \frac{-1}{\omega^{2}}\left(f\left(E_{f}\right)+n\left(E_{f}\right)\right) \\
= & -\frac{1}{4 \pi^{2} \omega}\left(\int_{0}^{\infty} d E_{f}-\int_{0}^{T^{*}} d E_{f}\right) \\
& \times E_{f}\left(f\left(E_{f}\right)+n\left(E_{f}\right)\right) . \quad(\mathrm{C} 16)
\end{aligned}
$$

Inserting Eq. (C8), we obtain

$$
\begin{aligned}
\operatorname{Re} \tilde{B}^{0}(\mathbf{0}, \omega ; m)_{T \neq 0}^{E_{f}>T^{*}} & \simeq-\frac{T^{2}}{16 \omega}+\frac{1}{4 \pi^{2} \omega} \int_{0}^{T^{*}} d E_{f} T \\
& =-\frac{T^{2}}{16 \omega}+\frac{T^{*} T}{4 \pi^{2} \omega} .
\end{aligned}
$$

The contribution from $E_{f}<T^{*}$ region, represented by $\operatorname{Re} \tilde{B}^{0}(\mathbf{0}, \omega ; m)_{T \neq 0}^{E_{f}<T^{*}}$, is estimated as follows by using Eq. (C8) and the inequality $\omega \gg m$ :

$$
\begin{aligned}
& \operatorname{Re} \tilde{B}^{0}(\mathbf{0}, \omega ; m)_{T \neq 0}^{E_{f}<T^{*}} \\
& \simeq \frac{\omega}{2 \pi^{2}} \mathrm{P} \int_{0}^{T^{*}} d E_{f} T \frac{E_{f}^{2}}{E_{f}^{2}+m^{2}} \frac{2 E_{f}^{2}-\omega^{2}}{\omega^{4}-4 E_{f}^{2} \omega^{2}} .
\end{aligned}
$$

Utilizing the inequalities $T \gg T^{*} \gg \omega$, $\operatorname{Re} \tilde{B}^{0}(\mathbf{0}, \omega ; m)_{T \neq 0}^{E_{f}<T^{*}}$ is evaluated as follows by utilizing partial fraction decomposition:

$$
\operatorname{Re} \tilde{B}^{0}(\mathbf{0}, \omega ; m)_{T \neq 0}^{E_{f}<T^{*}} \simeq-\frac{T T^{*}}{4 \pi^{2} \omega}-\frac{T}{4 \pi^{2} \omega}\left(-\pi m+\frac{\omega^{2}}{4 T^{*}}\right) .
$$

Since the largest term is $-T^{2} /(16 \omega)$, we have

$$
\operatorname{Re} \tilde{B}^{0}(\mathbf{0}, \omega ; m)_{T \neq 0} \simeq-\frac{T^{2}}{16 \omega},
$$

which is much larger than the imaginary part. A few remarks are in order here: the last formula leads to the wellknown result of the HTL approximation in QED. It is easy to understand if we remember that we can neglect $m$ and apply the HTL approximation in this situation, $T \gg \omega \gg m$.

From Eqs. (4.12) and (4.13), it seems that $T m /(4 \pi \omega)$ in Eq. (C19) yields terms which are of the order of $g^{2} T \omega / m$ and $g^{2} T \omega \sqrt{\alpha} / m$ in $\Sigma_{+}(\mathbf{0}, \omega)$, which implies that the massless limit $(m / T \rightarrow 0)$ cannot be taken. However, from Eq. (C14), we see that these terms are canceled by terms which come from Eqs. (4.8) and (4.9).

The results of this section, Eqs. (C12), (C14), (C17), and (C19), reproduce leading term of asymptotic form of some integrals used in [23] since the QED limit, $m / T, m / \omega \rightarrow 0$, is included in the limit considered in this section, $T \gg$ $\omega \gg m$. Results in other sections are not related to them because their situations are far from QED.

\section{2. $T \gg m \gg \omega$ \\ a. $\tilde{B}(\mathbf{0}, \omega ; m)$}

The imaginary part of $\tilde{B}(\mathbf{0}, \omega ; m)_{T \neq 0}$ is given by Eq. (C5), which is estimated as follows:

$$
\begin{aligned}
\operatorname{Im} \tilde{B}(\mathbf{0}, \omega ; m)_{T \neq 0} & \simeq \frac{-m^{2}}{16 \pi \omega^{2}}\left(f\left(\frac{m^{2}}{2 \omega}\right)+n\left(\frac{m^{2}}{2 \omega}\right)\right) \\
& =\frac{-m^{2}}{8 \pi \omega^{2}} \exp \left(\frac{m^{2}}{2 \omega T}\right) n\left(\frac{m^{2}}{\omega}\right) \\
& \simeq \begin{cases}\frac{-m^{2}}{8 \pi \omega^{2}} \exp \left(-\frac{m^{2}}{2 \omega T}\right) & \left(m^{2} \gg \omega T \text { case }\right) \\
-\frac{T}{8 \pi \omega} & \left(m^{2} \ll \omega T \text { case }\right) .\end{cases}
\end{aligned}
$$

In the first line the inequality $m \gg \omega$ has been taken into account, while in the second line, either $m^{2} \gg \omega T$ or $m^{2} \ll \omega T$ has been used.

Next, we estimate the real part by separating the cases $m^{2} \gg \omega T$ and $\omega T \gg m^{2}$.

(i) $m^{2} \gg \omega T$ case

We begin with Eq. (C3). We again introduce an intermediate scale $T^{*}$ satisfying $T \gg T^{*} \gg m$. The contribution from $E_{f}>T^{*}$ is evaluated as follows:

$$
\begin{aligned}
& \operatorname{Re} \tilde{B}(\mathbf{0}, \omega ; m)_{T \neq 0}^{E_{f}>T^{*}} \\
& \simeq-\frac{1}{2 \pi^{2} m^{2}} \int_{T^{*}}^{\infty} d E_{f} E_{f}\left(f\left(E_{f}\right)+n\left(E_{f}\right)\right),
\end{aligned}
$$

where the relation $E_{b} \simeq E_{f} \gg m \gg \omega$ has been utilized and the contribution from $E_{f} \gtrsim m^{2} / \omega$ has been neglected because it is suppressed by the Boltzmann factor $\sim \exp \left(-m^{2} /(\omega T)\right)$. Dividing the interval of 
the integral as in Eq. (C16), the following expression is obtained:

$$
\begin{aligned}
& \operatorname{Re} \tilde{B}(\mathbf{0}, \omega ; m)_{T \neq 0}^{E_{f}>T^{*}} \\
& \simeq-\frac{1}{2 \pi^{2} m^{2}}\left(\int_{0}^{\infty} d E_{f}-\int_{0}^{T^{*}} d E_{f}\right) E_{f}\left(f\left(E_{f}\right)+n\left(E_{f}\right)\right) \\
& \simeq-\frac{T^{2}}{8 m^{2}}+\frac{T^{*} T}{2 \pi^{2} m^{2}} .
\end{aligned}
$$

In the last line, we have inserted Eq. (C8). On the other hand, using Eq. (C8) and $m \gg \omega$, the contribution from $E_{f}<T^{*}$ is estimated to be

$$
\begin{aligned}
\operatorname{Re} \tilde{B}(\mathbf{0}, \omega ; m)_{T \neq 0}^{E_{f}<T^{*}} \simeq & -\frac{m^{2} T}{2 \pi^{2}} \mathrm{P} \int_{0}^{T^{*}} d E_{f} \frac{E_{f}^{2}}{E_{f}^{2}+m^{2}} \\
& \times \frac{1}{m^{4}-4 E_{f}^{2} \omega^{2}} .
\end{aligned}
$$

Utilizing the inequalities $T^{*} \ll m^{2} / \omega$ and $m \gg \omega$, we obtain

$$
\operatorname{Re} \tilde{B}(\mathbf{0}, \omega ; m)_{T \neq 0}^{E_{f}<T^{*}} \simeq-\frac{T T^{*}}{2 \pi^{2} m^{2}}+\frac{T}{4 \pi m},
$$

which is obtained by partial fraction decomposition. Taking only the largest term, we have

$$
\operatorname{Re} \tilde{B}(\mathbf{0}, \omega ; m)_{T \neq 0} \simeq-\frac{T^{2}}{8 m^{2}},
$$

which dominates the imaginary part.

(ii) $m^{2} \ll \omega T$ case

We introduce $T^{*}$, which is an intermediate scale satisfying $T \gg T^{*} \gg m^{2} / \omega$. From Eq. (C3), we have for the contribution from $E_{f}>T^{*}$

$$
\begin{aligned}
& \operatorname{Re} \tilde{B}(\mathbf{0}, \omega ; m)_{T \neq 0}^{E_{f}>T^{*}} \\
& \simeq \frac{m^{2}}{8 \pi^{2} \omega^{2}} \int_{T^{*}}^{\infty} d E_{f} \frac{1}{E_{f}}\left(f\left(E_{f}\right)+n\left(E_{f}\right)\right) \\
& \simeq \frac{m^{2} T}{8 \pi^{2} \omega^{2} T^{*}} .
\end{aligned}
$$

In the first line, we have used the inequalities $m \gg$ $\omega$ and $E_{b} \simeq E_{f}>T^{*} \gg m^{2} / \omega$, while in the second line an approximation similar to that after Eq. (C11) has been utilized.

Substituting Eq. (C8), the contribution from $E_{f}<$ $T^{*}$ is estimated to be

$$
\begin{aligned}
& \operatorname{Re} \tilde{B}(\mathbf{0}, \omega ; m)_{T \neq 0}^{E_{f}<T^{*}} \\
& \simeq-\frac{m^{2} T}{2 \pi^{2}} \mathrm{P} \int_{0}^{T^{*}} d E_{f} \frac{E_{f}^{2}}{E_{f}^{2}+m^{2}} \frac{1}{m^{4}-4 E_{f}^{2} \omega^{2}} \\
& \simeq-\frac{m^{2} T}{8 \pi^{2} \omega^{2} T^{*}}+\frac{T}{4 \pi m} .
\end{aligned}
$$

In the second line the inequality $T^{*} \gg m^{2} / \omega$ was used. Now we see that every term in the real part is much smaller than the imaginary part. Combining these results, we have

$$
\tilde{B}(\mathbf{0}, \omega ; m)_{T \neq 0} \simeq \begin{cases}-\frac{1}{8}\left(\frac{T}{m}\right)^{2} & \left(m^{2} \gg \omega T \text { case }\right) \\ -\frac{i}{8 \pi} \frac{T}{\omega} & \left(m^{2} \ll \omega T \text { case }\right) .\end{cases}
$$

$$
\text { b. } \tilde{B}^{0}(\mathbf{0}, \omega ; m)
$$

First, let us estimate the imaginary part of $\tilde{B}^{0}(\mathbf{0}, \omega ; m)_{T \neq 0}$ given by Eq. (C6):

$$
\begin{aligned}
& \operatorname{Im} \tilde{B}^{0}(\mathbf{0}, \omega ; m)_{T \neq 0} \\
& \simeq \begin{cases}\frac{m^{4}}{16 \pi \omega^{3}} \exp \left(-\frac{m^{2}}{2 \omega T}\right) & \left(m^{2} \gg \omega T \text { case }\right) \\
\frac{m^{2} T}{16 \pi \omega^{2}} & \left(m^{2} \ll \omega T \text { case }\right) .\end{cases}
\end{aligned}
$$

We have arrived at this expression in the same way as in Eq. (C21).

(i) $m^{2} \gg \omega T$ case

In this case, the real part of $\tilde{B}^{0}(\mathbf{p}, \omega ; m)_{T \neq 0}$ which is given by Eq. (C4) is evaluated as follows. Using an intermediate scale $T^{*}$ satisfying $T \gg T^{*} \gg m$, we have for the contribution from $E_{f}>T^{*}$

$$
\begin{aligned}
& \operatorname{Re} \tilde{B}^{0}(\mathbf{0}, \omega ; m)_{T \neq 0}^{E_{f}>T^{*}} \\
& \simeq \frac{\omega}{\pi^{2} m^{4}} \int_{T^{*}}^{\infty} d E_{f} E_{f}^{3}\left(f\left(E_{f}\right)+n\left(E_{f}\right)\right),
\end{aligned}
$$

where the contribution from $E_{f} \gtrsim m^{2} / \omega$ has been neglected since it is suppressed by the Boltzmann factor $\sim \exp \left(-m^{2} /(\omega T)\right)$. Also the inequality $m \gg \omega$ has been taken into account. Dividing the interval of the integral as in Eq. (C16), we obtain

$$
\begin{aligned}
& \operatorname{Re} \tilde{B}^{0}(\mathbf{0}, \omega ; m)_{T \neq 0}^{E_{f}>T^{*}} \\
& \simeq \frac{\omega}{\pi^{2} m^{4}}\left(\int_{0}^{\infty} d E_{f}-\int_{0}^{T^{*}} d E_{f}\right) E_{f}^{3}\left(f\left(E_{f}\right)+n\left(E_{f}\right)\right) \\
& \simeq \frac{\pi^{2}}{8} \frac{\omega T^{4}}{m^{4}}-\frac{\omega T T^{* 3}}{3 \pi^{2} m^{4}} .
\end{aligned}
$$

In the last line, we have substituted Eq. (C8).

Next, we estimate the contribution from $E_{f}<T^{*}$. Since the leading terms cancel out, we retain $\omega^{2}$ in Eq. (C4). If we define $\Omega_{ \pm}^{2} \equiv \omega^{2} \pm m^{2}$, the contribution from $E_{f}<T^{*}$ is estimated as follows:

$$
\begin{aligned}
\operatorname{Re} \tilde{B}^{0}(\mathbf{0}, \omega ; m)_{T \neq 0}^{E_{f}<T^{*}} \simeq & \frac{\omega T}{2 \pi^{2}} \mathrm{P} \int_{0}^{T^{*}} d E_{f} \frac{E_{f}^{2}}{E_{f}^{2}+m^{2}} \\
& \times \frac{2 E_{f}^{2}-\Omega_{-}^{2}}{\Omega_{-}^{4}-4 E_{f}^{2} \omega^{2}},
\end{aligned}
$$




\section{SPECTRAL FUNCTION OF A FERMION COUPLED WITH A ...}

where we have used Eq. (C8). Performing partial fraction decomposition, we have

$$
\begin{aligned}
& \operatorname{Re} \tilde{B}^{0}(\mathbf{0}, \omega ; m)_{T \neq 0}^{E_{f}<T^{*}} \\
& \simeq-\frac{T}{4 \pi^{2} \omega} \mathrm{P} \int_{0}^{T^{*}} d E_{f}\left(1-\frac{m^{2}}{E_{f}^{2}+m^{2}} \frac{2 \omega^{2}}{\Omega_{+}^{2}}\right. \\
& \left.\quad-\frac{\Omega_{-}^{6}}{4 \omega^{2} \Omega_{+}^{2}} \frac{1}{E_{f}^{2}-\Omega_{-}^{4} /\left(4 \omega^{2}\right)}\right) \\
& \simeq-\frac{T}{4 \pi^{2} \omega}\left(-\frac{\pi \omega^{2}}{m}+T^{*} \frac{2 \omega^{2}}{\Omega_{+}^{2}}+\frac{4}{3} \frac{\omega^{2} T^{* 3}}{\Omega_{+}^{2} \Omega_{-}^{2}}\right) .
\end{aligned}
$$

In the second line the inequality $T^{*} \gg m$ has been utilized. Notice that because of canceling out of the leading terms, we have to retain the next-to-leading term in the expansion of $\ln \left(1+2 \omega T^{*} / \Omega_{-}^{2}\right)$. The largest term is the first term of Eq. (C32), so

$$
\operatorname{Re} \tilde{B}^{0}(\mathbf{0}, \omega ; m)_{T \neq 0} \simeq \frac{\pi^{2}}{8} \frac{\omega T^{4}}{m^{4}},
$$

which is found to be much larger than the imaginary part.

(ii) $m^{2} \ll \omega T$ case

The real part is given by Eq. (C4). As is before, we introduce an intermediate scale $T^{*}$ satisfying $T \gg$ $T^{*} \gg m^{2} / \omega \gg m$. Then

$$
\operatorname{Re} \tilde{B}^{0}(\mathbf{0}, \omega ; m)_{T \neq 0}^{E_{f}>T^{*}} \simeq-\frac{T^{2}}{16 \omega}+\frac{T^{*} T}{4 \pi^{2} \omega} .
$$

This expression has been obtained in the same procedure as in Eqs. (C16) and (C17).

The contribution from $E_{f}<T^{*}$ is estimated as follows. Using Eq. (C8) and the inequality $m \gg \omega$, we have

$$
\begin{aligned}
\operatorname{Re} \tilde{B}^{0}(\mathbf{0}, \omega ; m)_{T \neq 0}^{E_{f}<T^{*}} \simeq & \frac{\omega}{2 \pi^{2}} \mathrm{P} \int_{0}^{T^{*}} d E_{f} T \frac{E_{f}^{2}}{E_{f}^{2}+m^{2}} \\
& \times \frac{2 E_{f}^{2}+m^{2}}{m^{4}-4 E_{f}^{2} \omega^{2}} \\
\simeq & -\frac{T T^{*}}{4 \pi^{2} \omega}+\frac{m^{4} T}{16 \pi^{2} \omega^{3} T^{*}} .
\end{aligned}
$$

In the last line we have utilized the inequality $T^{*} \gg$ $m^{2} / \omega \gg m$. Since $T^{*} \gg m^{4} /\left(\omega^{2} T\right)$, the largest term is found to be $-T^{2} /(16 \omega)$, so we have

$$
\operatorname{Re} \tilde{B}^{0}(\mathbf{0}, \omega ; m)_{T \neq 0} \simeq-\frac{T^{2}}{16 \omega} .
$$

This is much larger than the imaginary part.

Combining these results, we have

$$
\tilde{B}^{0}(\mathbf{0}, \omega ; m)_{T \neq 0} \simeq \begin{cases}\frac{\pi^{2}}{8} \frac{\omega T^{4}}{m^{4}} & \left(m^{2} \gg \omega T \text { case }\right) \\ -\frac{T^{2}}{16 \omega} & \left(m^{2} \ll \omega T \text { case }\right) .\end{cases}
$$

\section{3. $m \gg T \gg \omega$}

a. $\tilde{B}(\mathbf{0}, \omega ; m)$

We start with an estimate of the imaginary part of $\tilde{B}(\mathbf{0}, \omega ; m)_{T \neq 0}$ given by Eq. (C5). For $m \gg T \gg \omega$, we have

$$
\operatorname{Im} \tilde{B}(\mathbf{0}, \omega ; m)_{T \neq 0} \simeq-\frac{m^{2}}{8 \pi \omega^{2}} \exp \left(-\frac{m^{2}}{2 \omega T}\right) .
$$

The derivation of this expression is almost the same as that of Eq. (C21) in the $m^{2} \gg \omega T$ case. The real part of $\tilde{B}(\mathbf{0}, \omega ; m)_{T \neq 0}$ given by Eq. (C3) is estimated as follows:

$$
\operatorname{Re} \tilde{B}(\mathbf{0}, \omega ; m)_{T \neq 0} \simeq \frac{1}{2 \pi^{2}} \mathrm{P} \int_{0}^{\infty} d E_{f} E_{f} f\left(E_{f}\right) \frac{-m^{2}}{m^{4}-4 E_{f}^{2} \omega^{2}} .
$$

Here we have used the inequality $m \gg \omega$ and $n\left(E_{b}\right)$ has been neglected because of the suppression factor by $\exp (-m / T)$. Notice that the contribution from $E_{f} \gtrsim$ $\mathrm{m}^{2} / \omega \gg T$ is negligible owing to the suppression factor by $f\left(E_{f}\right)$. Then, we see that the denominator of the integrand can be approximated as $m^{4}-4 E_{f}^{2} \omega^{2} \simeq m^{4}$. Thus, we have

$$
\begin{aligned}
\operatorname{Re} \tilde{B}(\mathbf{0}, \omega ; m)_{T \neq 0} & \simeq \frac{-1}{2 \pi^{2} m^{2}} \mathrm{P} \int_{0}^{\infty} d E_{f} E_{f} f\left(E_{f}\right) \\
& =-\frac{T^{2}}{24 m^{2}},
\end{aligned}
$$

which is found to dominate the imaginary part.

$$
\text { b. } \tilde{B}^{0}(\mathbf{0}, \omega ; m)
$$

We estimate the imaginary part of $\tilde{B}^{0}(\mathbf{0}, \omega ; m)_{T \neq 0}$, which is given by Eq. (C6). Utilizing the same procedure as in Eq. (C40), we have

$$
\operatorname{Im} \tilde{B}^{0}(\mathbf{0}, \omega ; m)_{T \neq 0} \simeq \frac{m^{4}}{16 \pi \omega^{3}} \exp \left(-\frac{m^{2}}{2 \omega T}\right) .
$$

The real part, which is given by Eq. (C4), is estimated as follows:

$$
\operatorname{Re} \tilde{B}^{0}(\mathbf{0}, \omega ; m)_{T \neq 0} \simeq \frac{\omega}{\pi^{2}} \mathrm{P} \int_{0}^{\infty} d E_{f} E_{f}^{3} f\left(E_{f}\right) \frac{1}{m^{4}-4 E_{f}^{2} \omega^{2}} .
$$

Here we have utilized the inequality $m \gg \omega$ and neglected $n\left(E_{b}\right)$ since its contribution is suppressed by the factor $\exp (-m / T)$. Following the procedure used in obtaining Eq. (C42), we have

$$
\begin{aligned}
\operatorname{Re} \tilde{B}^{0}(\mathbf{0}, \omega ; m)_{T \neq 0} & \simeq \frac{\omega}{\pi^{2} m^{4}} \int_{0}^{\infty} d E_{f} E_{f}^{3} f\left(E_{f}\right) \\
& =\frac{7 \pi^{2}}{120} \frac{\omega T^{4}}{m^{4}} .
\end{aligned}
$$

One sees that this term dominates $\operatorname{Im} \tilde{B}^{0}(\mathbf{0}, \omega ; m)_{T \neq 0}$. 
DAISUKE SATOW, YOSHIMASA HIDAKA, AND TEIJI KUNIHIRO

PHYSICAL REVIEW D 83, 045017 (2011)

$$
\begin{gathered}
\text { 4. } \boldsymbol{T} \gg \boldsymbol{\omega} \sim \boldsymbol{m} \\
\text { a. } \tilde{B}(\mathbf{0}, \omega ; m)
\end{gathered}
$$

We start with an estimate of the imaginary part of $\tilde{B}(\mathbf{0}, \omega ; m)_{T \neq 0}$, which is given by Eq. (C5). Utilizing the inequality $T \gg \omega \sim m$ and substituting Eq. (C8), we have

$$
\operatorname{Im} \tilde{B}(\mathbf{0}, \omega ; m)_{T \neq 0} \simeq \frac{T}{8 \pi \omega} \frac{\omega^{2}-m^{2}}{\omega^{2}+m^{2}} \sim \frac{T}{\omega} .
$$

In the evaluation of the real part, we introduce $T^{*}$ satisfying $T \gg T^{*} \gg \omega$. From Eq. (C3), we arrive at the following expression by using the inequalities $E_{b} \sim E_{f} \gg \omega, m$ :

$\operatorname{Re} \tilde{B}(\mathbf{0}, \omega ; m)_{T \neq 0}^{E_{f}>T^{*}}$

$$
\begin{aligned}
& \simeq-\frac{1}{8 \pi^{2} \omega^{2}} \mathrm{P} \int_{T^{*}}^{\infty} d E_{f} \frac{1}{E_{f}}\left(\Omega_{-}^{2} f\left(E_{f}\right)-\Omega_{+}^{2} n\left(E_{f}\right)\right) \\
& \simeq \frac{\Omega_{+}^{2}}{8 \pi^{2} \omega^{2}} \frac{T}{T^{*}},
\end{aligned}
$$

with $\Omega_{ \pm}^{2} \equiv \omega^{2} \pm m^{2}$. In the last line we have performed the partial integration and the order estimate as we did for Eq. (C11).

Inserting Eq. (C8), the contribution from $E_{f}<T^{*}$ is estimated to be

$$
\begin{aligned}
\operatorname{Re} \tilde{B}(\mathbf{0}, \omega ; m)_{T \neq 0}^{E_{f}<T^{*}} \simeq & \frac{T \Omega_{+}^{2}}{8 \pi^{2} \omega^{2}} \mathrm{P} \int_{0}^{T^{*}} d E_{f} \frac{E_{f}^{2}}{E_{f}^{2}+m^{2}} \\
& \times \frac{1}{E_{f}^{2}-\Omega_{-}^{4} /\left(4 \omega^{2}\right)} .
\end{aligned}
$$

Using the inequality $T \gg T^{*} \gg \omega \sim m$ and carrying out partial fraction decomposition, the following estimation is obtained:

$$
\operatorname{Re} \tilde{B}(\mathbf{0}, \omega ; m)_{T \neq 0}^{E_{f}<T^{*}} \simeq-\frac{T}{8 \pi^{2} \omega^{2}} \frac{\Omega_{-}^{4}}{\Omega_{+}^{2} T^{*}}+\frac{m}{\Omega_{+}^{2}} \frac{T}{4 \pi} \sim \frac{T}{\omega} .
$$

We see that both of the real part and the imaginary part are of the order of $T / \omega$;

$$
\tilde{B}(\mathbf{0}, \omega ; m)_{T \neq 0} \sim \frac{T}{\omega} .
$$

$$
\text { b. } \tilde{B}^{0}(\mathbf{0}, \omega ; m)
$$

We make an order estimate of the imaginary part of $\tilde{B}^{0}(\mathbf{0}, \omega ; m)_{T \neq 0}$ given by Eq. (C6). Repeating the same derivation leading to Eq. (C46), we have

$$
\operatorname{Im} \tilde{B}^{0}(\mathbf{0}, \omega ; m)_{T \neq 0} \simeq \frac{T}{16 \pi \omega^{2}} \frac{\left(m^{2}-\omega^{2}\right)^{2}}{m^{2}+\omega^{2}} \sim T .
$$

From Eq. (C4), we estimate the contribution from the $E_{f}>T^{*}$ region to its real part as follows:

$$
\operatorname{Re} \tilde{B}^{0}(\mathbf{0}, \omega ; m)_{T \neq 0}^{E_{f}>T^{*}} \simeq-\frac{T^{2}}{16 \omega}+\frac{T^{*} T}{4 \pi^{2} \omega} .
$$

Here we have introduced $T^{*}$ which satisfies $T \gg T^{*} \gg m$, and used the same approximations as that adopted in Eqs. (C16) and (C17).

On the other hand, the contribution from $E_{f}<T^{*}$ is estimated as follows by using Eq. (C8):

$\operatorname{Re} \tilde{B}^{0}(\mathbf{0}, \omega ; m)_{T \neq 0}^{E_{f}<T^{*}}$

$$
\begin{aligned}
\simeq & \frac{\omega}{2 \pi^{2}} \mathrm{P} \int_{0}^{T^{*}} d E_{f} T \frac{E_{f}^{2}}{E_{f}^{2}+m^{2}} \frac{2 E_{f}^{2}-\Omega_{-}^{2}}{\Omega_{-}^{4}-4 E_{f}^{2} \omega^{2}} \\
\simeq & -\frac{T}{4 \pi^{2} \omega}\left(T^{*}-m \frac{\pi}{2}+\frac{\Omega_{-}^{2}}{2}\left(\frac{\Omega_{-}^{2}}{2 \omega^{2}}-1\right)\left(-\frac{1}{T^{*}}\right)\right. \\
& \left.+m^{2} \Omega_{-}^{2} \frac{\Omega_{-}^{2}-2 \omega^{2}}{\Omega_{-}^{4}+4 m^{2} \omega^{2}}\left(m \frac{\pi}{2}+\frac{1}{T^{*}}\right)\right),
\end{aligned}
$$

with $\Omega_{-}^{2} \equiv \omega^{2}-m^{2}$. In the last line, the inequality $T^{*} \gg$ $\omega \sim m$ has been utilized and partial fraction decomposition has been performed. Since the largest term is $-T^{2} /(16 \omega)$ in Eq. (C52), we have

$$
\operatorname{Re} \tilde{B}^{0}(\mathbf{0}, \omega ; m)_{T \neq 0} \simeq-\frac{T^{2}}{16 \omega},
$$

which is much larger than the imaginary part.
[1] J. Frenkel and J. C. Taylor, Nucl. Phys. B334, 199 (1990).

[2] E. Braaten and R.D. Pisarski, Nucl. Phys. B337, 569 (1990); B339, 310 (1990).

[3] H. A. Weldon, Phys. Rev. D 26, 1394 (1982).

[4] H. A. Weldon, Phys. Rev. D 26, 2789 (1982); 40, 2410 (1989).

[5] M. Kitazawa, T. Kunihiro, and Y. Nemoto, Phys. Lett. B 633, 269 (2006).

[6] Y. Nambu and G. Jona-Lasinio, Phys. Rev. 122, 345 (1961); 124, 246 (1961); As a review article, see
T. Hatsuda and T. Kunihiro, Phys. Rep. 247, 221 (1994).

[7] T. Hatsuda and T. Kunihiro, Phys. Rev. Lett. 55, 158 (1985).

[8] M. Kitazawa, T. Kunihiro, and Y. Nemoto, Prog. Theor. Phys. 117, 103 (2007).

[9] M. Kitazawa, T. Kunihiro, K. Mitsutani, and Y. Nemoto, Phys. Rev. D 77, 045034 (2008).

[10] E. C. G. Stueckelberg, Helv. Phys. Acta 11, 225 (1938); 11, 299 (1938); 11, 312 (1938). 


\section{SPECTRAL FUNCTION OF A FERMION COUPLED WITH A ...}

PHYSICAL REVIEW D 83, 045017 (2011)

[11] As a review article, see H. Ruegg and M. Ruiz-Altaba, Int. J. Mod. Phys. A 19, 3265 (2004).

[12] R. Kobes, G. Kunstatter, and A. Rebhan, Phys. Rev. Lett. 64, 2992 (1990); Nucl. Phys. B355, 1 (1991); A. Rebhan, Lect. Notes Phys. 583, 161 (2002).

[13] G. E. Brown and M. Rho, Phys. Rev. Lett. 66, 2720 (1991); T. Kunihiro, Nucl. Phys. B351, 593 (1991); T. Hatsuda, Y. Koike, and S. H. Lee, Nucl. Phys. B394, 221 (1993); M. Harada and K. Yamawaki, Phys. Rev. Lett. 86, 757 (2001); Y. Hidaka, O. Morimatsu, and M. Ohtani, Phys. Rev. D 73, 036004 (2006); M. Harada and C. Sasaki, Phys. Lett. B 537, 280 (2002); Phys. Rev. D 73, 036001 (2006).

[14] D. Notzold and G. Raffelt, Nucl. Phys. B307, 924 (1988); G. F. Giudice, A. Notari, M. Raidal, A. Riotto, and A. Strumia, ibid. B685, 89 (2004); D. Boyanovsky, Phys. Rev. D 72, 033004 (2005).

[15] M. Le Bellac, Thermal Field Theory (Cambridge University Press, Cambridge, UK, 1996).

[16] J. I. Kapusta and C. Gale, Finite-Temperature Field Theory: Principles and Applications (Cambridge University Press, Cambridge, UK, 2006).

[17] T. W. B. Kibble, in Proceedings of the Oxford International Conference on Elementary Particles (Rutherford High Energy Laboratory, Harwell, England, 1965), p. 19.
[18] S. Weinberg, Phys. Rev. D 9, 3357 (1974).

[19] L. Dolan and R. Jackiw, Phys. Rev. D 9, 3320 (1974).

[20] V. V. Lebedev and A. V. Smilga, Ann. Phys. (N.Y.) 202, 229 (1990); see also S. Jeon, Phys. Rev. D 52, 3591 (1995); J.S. Gagnon and S. Jeon, Phys. Rev. D 75, 025014 (2007); 76, 089902 (2007); J. S. Gagnon and S. Jeon, ibid.76, 105019 (2007); Y. Hidaka and T. Kunihiro, arXiv:1009.5154.

[21] Y. Hidaka, D. Satow, and T. Kunihiro (unpublished).

[22] S. Y. Wang, Phys. Rev. D 70, 065011 (2004).

[23] E. Mottola and Z. Szep, Phys. Rev. D 81, 025014 (2010).

[24] C. DeTar, Phys. Rev. D 32, 276 (1985); C. DeTar and J. Kogut, Phys. Rev. Lett. 59, 399 (1987).

[25] E. V. Shuryak and I. Zahed, Phys. Rev. C 70, 021901 (2004).

[26] S. Datta, F. Karsch, P. Petreczky, and I. Wetzorke, Nucl. Phys. B, Proc. Suppl. 119, 487 (2003); Phys. Rev. D 69 , 094507 (2004); T. Umeda, H. Matsufuru, O. Miyamura, and K. Nomura, Nucl. Phys. A721, C922 (2003); T. Umeda, K. Nomura, and H. Matsufuru, Eur. Phys. J. C 39, 9 (2004); M. Asakawa and T. Hatsuda, Phys. Rev. Lett. 92, 012001 (2004); 